\title{
Stochastic Properties of Generalized Finite $\alpha$-Mixtures
}

\author{
Omid Shojaee, $\quad$ Majid Asadi, ${ }^{\dagger}$ \& $\quad$ Maxim Finkelstein ${ }^{\ddagger}$
}

\begin{abstract}
Most of real-life populations are heterogeneous and homogeneity is often just a simplifying assumption for the relevant statistical analysis. Mixtures of lifetime distributions that correspond to homogeneous sub-populations were intensively studied in the literature. Various distributional and stochastic properties of finite and continuous mixtures were discussed. In this paper, following recent publications, we develop further a mixture concept in the form of the generalized alpha mixtures that include all mixture models that are widely explored in the literature. We study some main stochastic properties of the suggested mixture model, i.e., aging and appropriate stochastic comparisons. Some relevant examples and counter-examples are given to illustrate our findings.
\end{abstract}

Keywords: Finite mixtures; Additive mixture model; Multiplicative mixture model; Stochastic orders; Majorization.

\section{Introduction}

\subsection{Motivation and related literature}

It is hard to find homogeneous populations in real life, as most populations of items in practice are non-homogeneous. Heterogeneous populations in reality consist usually from a finite number of homogeneous populations. For example, populations of manufactured items in practice often have two aggregated groups, i.e., the 'defective' items with shorter lifetimes and 'standard' items with longer, normal lifetimes (Block et al., 2003). Heterogeneity also occurs when components manufactured at different facilities are mixed, or they are manufactured at the same facility but with changing operational or environmental characteristics, etc (Finkelstein, 2008; Cha and Finkelstein, 2013). Ignoring inherent heterogeneity in populations of items can lead to fundamental errors in the corresponding reliability analysis. Finite mixture models are usually an effective tool for modeling heterogeneity. Some applications of finite mixture models can be found, for example, in Everitt and Hand (1981).

Let $\bar{F}(t)$ denote the survival function (SF) that describes the lifetime of an ordinary (arithmetic) finite mixture of $n$ homogeneous sub-populations with the SF's $\bar{F}_{i}(t), i=1, \ldots, n$. Then

\footnotetext{
${ }^{*}$ Department of Statistics, University of Isfahan, Isfahan 81744, Iran (E-mail: O.shojaee@sci.ui.ac.ir)

${ }^{\dagger}$ Department of Statistics, University of Isfahan, Isfahan 81744, Iran \& School of Mathematics, Institute for Research in Fundamental Sciences (IPM), P.O Box 19395-5746, Tehran, Iran (E-mail: m.asadi@sci.ui.ac.ir)

${ }^{\ddagger}$ Department of Mathematical Statistics, University of the Free State, 339 Bloemfontein 9300, South Africa \& Department of Management Science, University of Strathclyde, Glasgow, UK (E-mail: Finkelm@ufs.ac.za)
} 


$$
\bar{F}(t)=\sum_{i=1}^{n} p_{i} \bar{F}_{i}(t),
$$

where $p_{i}$ is the mixing proportion such that $\sum_{i=1}^{n} p_{i}=1$ and $p_{i} \geq 0$, for $i \in\{1,2, \ldots, n\}$.

Many authors have explored various aspects of mixture models. For instance, the tail behavior of the corresponding failure rates in mixture models has been investigated by Block et al. (1993) and Block and Joe (1997). Preservation of relevant stochastic properties for mixtures has been examined by Block et al. (2003). Block et al. (2003) also have studied mixtures with increasing linear failure rates. Badia et al. (2001) have provided bounds for the failure rate, the failure rate average, the mean residual life and their derivatives. The relevant aging properties of the additive and proportional (multiplicative) hazards mixing models have been investigated by Badia et al. (2002). Aging properties for the multivariate proportional hazards in mixtures as well as relevant stochastic comparisons have been studied by Badia and Lee (2020). Likelihood ratio ordering in the mixture models using the Glaser's function has been investigated by Navarro (2008). Stochastic comparisons for general mixtures in the sense of the hazard rate order and the likelihood ratio order have been studied in Navarro (2016). Navarro and Aguila (2017) have obtained the necessary and sufficient conditions to compare two finite mixture models in the sense of the usual stochastic order and the hazard rate order. Stochastic comparisons for two finite mixture models with different baseline random variables and different mixing proportions have been studied by Amini and Zhang (2017).

Hazra and Finkelstein (2018), using the multivariate majorization, have provided a stochastic comparison for two finite mixtures when the baseline distribution belongs to some semi-parametric families of distribution such as proportional hazards, accelerated lifetimes (scale models) and proportional reversed hazards models. Stochastic comparison for two finite mixtures using majorization in the sense of usual stochastic order, hazard rate order and reversed hazard rate order have been discussed by Nadeb and Torabi (2020). Albabtain et al. (2020) have considered a parametric family of weighted distributions and their mixtures and have provided some stochastic comparisons. For some aging properties and stochastic comparisons of mixtures, we refer, among others, to Finkelstein and Esaulova (2001), Finkelstein and Esaulova (2006), Shaked and Shanthikumar (2007) and Misra and Naqvi (2018).

Recently, Asadi et al. (2019) have proposed a flexible mixture model; the, so called, $\alpha$-mixture model that combines two popular in applications mixture models, i.e., the ordinary (arithmetic) mixture model and the mixture of failure rates model. In accordance with this paper, the finite $\alpha$-mixture for $n$ sub-populations with the corresponding SF's $\bar{F}_{i}, i=1,2, . ., n$, is defined as

$$
\bar{F}_{\alpha}(t)= \begin{cases}{\left[\sum_{i=1}^{n} p_{i} \bar{F}_{i}^{\alpha}(t)\right]^{1 / \alpha},} & \alpha \neq 0 \\ \prod_{i=1}^{n} \bar{F}_{i}^{p_{i}}(t), & \alpha=0\end{cases}
$$

where $p_{i}$ is the mixing proportion such that $p_{i} \geq 0$, for $i=1, \ldots, n$ and $\sum_{i=1}^{n} p_{i}=1$. Thus (2), for $\alpha=1$ reduces to the arithmetic (ordinary) mixture of survival functions, whereas the case $\alpha \rightarrow 0$, as shown by Asadi et al. (2019), defines the mixture of the failure rates (the geometric mixture of the corresponding SF's). Asadi et al. (2019) have extended the classical result of Barlow and Proschan (1975) on DFR (DFRA) distributions in $\alpha$-mixtures stating that the $\alpha$-mixture of DFR (IFR) distributions are DFR (IFR) for $\alpha>0(\alpha<0)$ (see Section 1.2 for definitions of these notions of aging). These authors also have shown that this closure property holds for DFRA 
(IFRA) distributions for $\alpha>0(\alpha<0)$. Shojaee et al. (2021) have considered the $\alpha$-mixture model and provided a new interpretation for the $\alpha$-mixture model via the multiplicative-additive hazard transform.

An obvious shortcoming of the model in (2) is that the parameter $\alpha$ is the same for all survival functions, whereas the weights are different. For instance, an impact of environment on different components of a multi-component system can be also different. Obviously, (2) is the specific case of a more general and practically sound in various applications and settings model with different parameters (see our discussion and examples in the beginning of the next section). Therefore, in the current study, motivated by Asadi et al. (2019), we generalize some results of this paper on the finite $\alpha$-mixture model (e,g., relevant stochastic comparisons and aging properties) to the case with different parameters. We also discuss in detail the limiting behavior of resulting distributions, conditional characteristics and relevant stochastic ordering using majorization technique. The latter is the main focus of the second part of the paper, where some new approaches are developed and discussed using relevant examples and counterexamples.

\subsection{Notation}

For two random variables $X$ and $Y$, we denote by $f$ and $g$, the probability density functions, by $F$ and $G$, the cumulative distribution functions, by $\bar{F}$ and $\bar{G}$, the survival functions and by $r_{X}$ and $r_{Y}$, the hazard (failure) rate functions, respectively. In this study, we will use the following concepts of aging and definitions of stochastic orders:

- The random variable $X$ (or its distribution $F$ ) is said to be increasing (decreasing) failure rate (IFR (DFR)) if its failure rate $r_{X}(t)$ is non-decreasing (non-increasing) in $t$.

- The random variable $X$ (or its distribution $F$ ) is said to be increasing (decreasing) failure rate average (IFRA (DFRA)) if $-\frac{1}{t} \log \bar{F}(t)$ is non-decreasing (non-increasing) in $t$.

- The random variable $X$ is said to be smaller than $Y$ in the usual stochastic order (denoted by $X \leq_{s t} Y$ or $\left.F \leq_{s t} G\right)$ if $\bar{F}(t) \leq \bar{G}(t)$ for all $t$.

- The random variable $X$ is said to be smaller than $Y$ in the hazard rate order (denoted by $X \leq_{h r} Y$ or $\left.F \leq_{h r} G\right)$ if $\bar{G}(t) / \bar{F}(t)$ is increasing in $t$, for all $t$ or equivalently $r_{X}(t) \geq r_{Y}(t)$, for all $t$.

- The random variable $X$ is said to be smaller than $Y$ in the likelihood ratio order (denoted by $X \leq_{l r} Y$ or $\left.F \leq_{l r} G\right)$ if $g(t) / f(t)$ is increasing in $t$, for all $t$.

\subsection{Organization of the paper}

The organization of the paper is as follows. Section 2 defines the generalized finite $\alpha$-mixture model and discusses its relation to other models reported in the literature. Section 3 studies aging properties of the generalized finite $\alpha$-mixture. Section 4 is devoted to the special cases of additive and multiplicative models for the baseline distributions in the generalized finite $\alpha$-mixture. Section 5 provides some stochastic comparisons in the usual stochastic order and the hazard rate order with different baseline distributions. Section 6 provides sufficient conditions based on the concept 
of majorization for stochastic comparison of two generalized finite $\alpha$-mixtures. Finally, Section 7 gives some concluding remarks.

\section{$2 \quad$ The generalized finite $\alpha$-mixture model}

The generalized finite $\alpha$-mixture of $n$ sub-populations with SF's $\bar{F}_{i}, i=1,2, . ., n$, is defined as

$$
\bar{F}(t, \bar{\alpha})=\left[\sum_{i=1}^{n} p_{i} \bar{F}_{i}^{\alpha_{i}}(t)\right]^{1 / \bar{\alpha}}, \quad \alpha_{i} \in(-\infty, \infty),
$$

where, $p_{i}$ is the mixing proportion such that $p_{i} \geq 0$, for $i \in\{1,2, \ldots, n\}$ and $\sum_{i=1}^{n} p_{i}=1$, and $\bar{\alpha}=\sum_{i=1}^{n} p_{i} \alpha_{i}$. Assuming that $\boldsymbol{p}=\left(p_{1}, \ldots, p_{n}\right)$ is given, it includes the following special cases:

- $\alpha_{i}=1, i=1, \ldots, n$, give the arithmetic mixture of $\bar{F}_{i}$ 's.

- $\alpha_{i}=-1, i=1, \ldots, n$, give the harmonic mixture of $\bar{F}_{i}$ 's.

- If $\alpha_{i}=0, i=1, \ldots, n$, tend to 0 , we arrive at the geometric mixture of $\bar{F}_{i}$ 's.

- $\alpha_{i}=\alpha, i=1, \ldots, n$, give the $\alpha$-mixture of survival function.

\section{Example:}

Assume that we have a mixed population with two sub-populations. Let the survival functions of items in each sub-populations in laboratory conditions be denoted as $\bar{F}_{i}(t), i=1,2$. Suppose that the corresponding mixing proportions are $p$ and $(1-p)$, respectively. Assume that the severe conditions act on each sub-population so that the survival functions of $i$ th sub-population, in accordance with the proportional hazards model, becomes $\bar{F}_{i}^{\alpha_{i}}(t)$, where $\alpha_{i}>0, i=1,2$. Then, the survival function of a randomly selected item is

$$
\bar{F}_{s}(t, \boldsymbol{\alpha})=p \bar{F}_{1}^{\alpha_{1}}(t)+(1-p) \bar{F}_{2}^{\alpha_{2}}(t) .
$$

Assume now that an item is shielded from the severe conditions. However, this shielding is 'executed' also by the proportional hazards model that decreases proportionally the failure rate that corresponds to the foregoing survival function. Thus, as it can be clearly seen, by this operation, we cannot arrive exactly at the initial mixed survival function (in laboratory conditions). Since the severe conditions parameter of the first selected item is $\alpha_{1}$ with probability $p$, and that of the second item is $\alpha_{2}$ with probability $(1-p)$, we consider the average value of the parameter in accordance with which the selected item is shielded, i.e., $p \alpha_{1}+(1-p) \alpha_{2}$. Hence, the survival function of the selected component after these operations is

$$
\bar{F}(t, \bar{\alpha})=\left(p \bar{F}_{1}^{\alpha_{1}}(t)+(1-p) \bar{F}_{2}^{\alpha_{2}}(t)\right)^{\frac{1}{\bar{\alpha}}},
$$

where $\bar{\alpha}=p \alpha_{1}+(1-p) \alpha_{2}$. 


\section{Relation to models in the literature}

In what follows, we interpret some of the existent in the literature specific models in terms of the generalized finite $\alpha$-mixtures. Note that the sub-populations/populations of items are considered as sufficiently large. Cha (2011) have considered the following two selection policies for designing the $m$-component series system:

- The $m$-component series systems are built only using one type of components. Assume that the proportion of systems built using the $i$-th component with the $\mathrm{SF} \bar{F}_{i}(t), i=1, \ldots, n$, is $p_{i}, i=1, \ldots, n$. A system is randomly selected from the large mixed set of systems. This model is defined as "mixing at the system level". The SF of the selected series system is

$$
\overline{\mathcal{F}}_{1}(t)=\sum_{i=1}^{n} p_{i} \bar{F}_{i}^{m}(t)=\bar{F}^{m}(t, m),
$$

where $\bar{F}(t, m)$, as the $m$-th root of the sum above, according to our definition, is the SF of the generalized finite $\alpha$-mixture with $\alpha_{i}=m, i=1, \ldots, n$. Let $\bar{F}_{i}(t)$ belong to the proportional hazard model, $\bar{F}_{i}(t)=\bar{G}_{i}^{r_{i}}(t)$ and set $m r_{i}=\alpha_{i}, i=1, \ldots, n$. Thus,

$$
\overline{\mathcal{F}}_{1}(t)=\sum_{i=1}^{n} p_{i} \bar{G}_{i}^{\alpha_{i}}(t)=\bar{G}^{\bar{\alpha}}(t, \bar{\alpha})=\bar{G}^{m \bar{r}}(t, m \bar{r}),
$$

where $\bar{G}(t, \bar{\alpha})$ is the SF of the generalized finite $\alpha$-mixture corresponding to $\bar{G}_{i}, i=1, \ldots, n$, and $\bar{r}=\sum_{i=1}^{n} p_{i} r_{i}$.

- The system is 'built randomly' using $n$ types of components. If the proportion of the $i$-th component with the SF $\bar{F}_{i}(t)$ is $p_{i}, i=1, \ldots, n$, then the SF of the built series system of $m$ components is:

$$
\overline{\mathcal{F}}_{2}(t)=\left(\sum_{i=1}^{n} p_{i} \bar{F}_{i}(t)\right)^{m}=\bar{F}^{m}(t, 1),
$$

where $\bar{F}(t, 1)$, is the $\mathrm{SF}$ of the generalized finite $\alpha$-mixture with $\alpha_{i}=1, i=1, \ldots, n$. This model can be called "mixing at the component level". Let $\bar{F}_{i}(t)$ belong to the proportional hazard model, $\bar{F}_{i}(t)=\bar{G}_{i}^{\alpha_{i}}(t), i=1, \ldots, n$. Thus,

$$
\overline{\mathcal{F}}_{2}(t)=\left(\sum_{i=1}^{n} p_{i} \bar{G}_{i}^{\alpha_{i}}(t)\right)^{m}=\left(\bar{G}^{\bar{\alpha}}(t, \bar{\alpha})\right)^{m},
$$

where $\bar{G}(t, \bar{\alpha})$ is the SF of the generalized finite $\alpha$-mixture corresponding to $\bar{G}_{i}, i=1, \ldots, n$, and $\bar{\alpha}=\sum_{i=1}^{n} p_{i} \alpha_{i}$.

For generalizations of these two models see Hazra et al. (2017). 


\section{Hazard rate and aging properties of the generalized finite $\alpha$ - mixture}

The generalized finite $\alpha$-mixture of SF's $\bar{F}_{i}$ for $i=1,2, . ., n$, in accordance with our definition, is

$$
\bar{F}(t, \bar{\alpha})=\left[\sum_{i=1}^{n} p_{i} \bar{F}_{i}^{\alpha_{i}}(t)\right]^{1 / \bar{\alpha}}, \quad \alpha_{i} \in(-\infty, \infty) .
$$

Then the corresponding PDF is:

$$
f(t, \bar{\alpha})=\frac{1}{\bar{\alpha}}\left[\sum_{i=1}^{n} \alpha_{i} p_{i} f_{i}(t) \bar{F}_{i}^{\alpha_{i}-1}(t)\right]\left[\sum_{i=1}^{n} p_{i} \bar{F}_{i}^{\alpha_{i}}(t)\right]^{\frac{1}{\bar{\alpha}-1}},
$$

where $p_{i}$ is the mixing proportion such that $\sum_{i=1}^{n} p_{i}=1$ and $p_{i} \geq 0$, for $i \in\{1,2, \ldots, n\}$ and $\bar{\alpha}=\sum_{i=1}^{n} p_{i} \alpha_{i}$.

If $r(t, \bar{\alpha})$ and $r_{i}(t)$ denote, respectively, the hazard rate of the generalized finite $\alpha$-mixture and the hazard rate of the $i-t h$ component, then

$$
\begin{aligned}
r(t, \bar{\alpha}) & =\frac{f(t, \bar{\alpha})}{\bar{F}(t, \bar{\alpha})} \\
& =\frac{1}{\bar{\alpha}} \frac{\sum_{i=1}^{n} \alpha_{i} p_{i} r_{i}(t) \bar{F}_{i}^{\alpha_{i}}(t)}{\sum_{j=1}^{n} p_{j} \bar{F}_{j}^{\alpha_{j}}(t)} \\
& =\frac{1}{\bar{\alpha}} \sum_{i=1}^{n} \alpha_{i} r_{i}(t) p_{\alpha_{i}}(t),
\end{aligned}
$$

where $p_{\alpha_{i}}(t)=\frac{p_{i} \bar{F}_{i}^{\alpha_{i}}(t)}{\sum_{j=1}^{n} p_{j} \bar{F}_{j}^{\alpha_{j}}(t)}$.

As an example, let us consider first, a mixture of two SFs $\bar{F}_{1}(t)$ and $\bar{F}_{2}(t)$ with PDFs $f_{1}(t)$ and $f_{2}(t)$ and hazard rates $r_{1}(t)$ and $r_{2}(t)$, respectively. We are interested in the bounds for the mixture hazard rate that is defined in this case as

$$
\begin{aligned}
r(t, \bar{\alpha}) & =\frac{1}{\bar{\alpha}}\left[\alpha_{1} r_{1}(t) \frac{p \bar{F}_{1}^{\alpha_{1}}(t)}{p \bar{F}_{1}^{\alpha_{1}}(t)+(1-p) \bar{F}_{2}^{\alpha_{2}}(t)}+\alpha_{2} r_{2}(t) \frac{(1-p) \bar{F}_{2}^{\alpha_{2}}(t)}{p \bar{F}_{1}^{\alpha_{1}}(t)+(1-p) \bar{F}_{2}^{\alpha_{2}}(t)}\right] \\
& =\frac{1}{\bar{\alpha}}\left[\alpha_{1} r_{1}(t) p_{\alpha}(t)+\alpha_{2} r_{2}(t)\left(1-p_{\alpha}(t)\right)\right],
\end{aligned}
$$

where the time-dependent probabilities are

$$
p_{\alpha}(t)=\frac{p \bar{F}_{1}^{\alpha_{1}}(t)}{p \bar{F}_{1}^{\alpha_{1}}(t)+(1-p) \bar{F}_{2}^{\alpha_{2}}(t)}, \quad\left(1-p_{\alpha}(t)\right)=\frac{(1-p) \bar{F}_{2}^{\alpha_{2}}(t)}{p \bar{F}_{1}^{\alpha_{1}}(t)+(1-p) \bar{F}_{2}^{\alpha_{2}}(t)} .
$$

From this representation we have (Finkelstein, 2008):

$$
\min \left\{\frac{\alpha_{1}}{\bar{\alpha}} r_{1}(t), \frac{\alpha_{2}}{\bar{\alpha}} r_{2}(t)\right\} \leq r(t, \bar{\alpha}) \leq \max \left\{\frac{\alpha_{1}}{\bar{\alpha}} r_{1}(t), \frac{\alpha_{2}}{\bar{\alpha}} r_{2}(t)\right\}
$$


In particular for $\alpha_{i}>0$, if $\alpha_{1} \leq \alpha_{2}$ and $F_{1} \geq_{h r} F_{2}$, then

$$
\frac{\alpha_{1}}{\bar{\alpha}} r_{1}(t) \leq r(t, \bar{\alpha}) \leq \frac{\alpha_{2}}{\bar{\alpha}} r_{2}(t),
$$

and for $\alpha_{i}<0$, if $\alpha_{1} \leq \alpha_{2}$ and $F_{1} \leq_{h r} F_{2}$, then

$$
\frac{\alpha_{2}}{\bar{\alpha}} r_{2}(t) \leq r(t, \bar{\alpha}) \leq \frac{\alpha_{1}}{\bar{\alpha}} r_{1}(t) .
$$

Generalizing the foregoing result, in the following theorem, we show that the lifetimes in the weakest (strongest) sub-population is smaller (greater) than that of the generalized finite $\alpha$-mixture of $n$ sub-populations in the sense of the hazard rate order for $\alpha_{i}>0\left(\alpha_{i}<0\right), i=1,2, \ldots, n$.

Theorem 3.1. Let the lifetimes in sub-populations be ordered in the sense of the hazard rate ordering, i.e., $F_{1} \leq_{h r} F_{2} \leq_{h r} \cdots \leq_{h r} F_{n}$. Then, for all $t$,

$$
F_{1} \leq_{h r} F(t, \bar{\alpha}),
$$

for all ordered $\alpha_{i}>0, i=1, \ldots, n$, whereas for all ordered $\alpha_{i}<0, i=1, \ldots, n$ :

$$
F(t, \bar{\alpha}) \leq_{h r} F_{n} .
$$

Proof. We only give proof for $\alpha_{i}>0, i=1, \ldots, n$ because the proof for $\alpha_{i}<0, i=1, \ldots, n$ is completely similar. Let $\alpha_{i}>0, i=1, \ldots, n$. We need show that $r(t, \bar{\alpha})-r_{1}(t) \leq 0$ for all $t$. We have

$$
\begin{aligned}
r(t, \bar{\alpha})-r_{1}(t) & =\frac{1}{\bar{\alpha}\left(\sum_{i=1}^{n} p_{i} \bar{F}_{i}^{\alpha_{i}}(t)\right)}\left(\sum_{i=1}^{n} r_{i}(t) \alpha_{i} p_{i} \bar{F}_{i}^{\alpha_{i}}(t)\right)-r_{1}(t) \\
& =\frac{\left(\sum_{i=1}^{n} r_{i}(t) \alpha_{i} p_{i} \bar{F}_{i}^{\alpha_{i}}(t)\right)-\left(\left(\sum_{i=1}^{n} \alpha_{i} p_{i}\right)\left(\sum_{i=1}^{n} p_{i} \bar{F}_{i}^{\alpha_{i}}(t)\right)\right) r_{1}(t)}{\bar{\alpha}\left(\sum_{i=1}^{n} p_{i} \bar{F}_{i}^{\alpha_{i}}(t)\right)} \\
& \stackrel{\operatorname{sign}}{=}\left(\sum_{i=1}^{n} r_{i}(t) \alpha_{i} p_{i} \bar{F}_{i}^{\alpha_{i}}(t)\right)-\left(\left(\sum_{i=1}^{n} \alpha_{i} p_{i}\right)\left(\sum_{i=1}^{n} p_{i} \bar{F}_{i}^{\alpha_{i}}(t)\right)\right) r_{1}(t) .
\end{aligned}
$$

From the assumption $F_{1} \leq_{h r} F_{2} \leq_{h r} \cdots \leq_{h r} F_{n}$, we have $r_{1}(t) \geq \cdots \geq r_{n}(t)$. This implies that

$$
\begin{aligned}
& \left(\sum_{i=1}^{n} r_{i}(t) \alpha_{i} p_{i} \bar{F}_{i}^{\alpha_{i}}(t)\right)-\left(\left(\sum_{i=1}^{n} \alpha_{i} p_{i}\right)\left(\sum_{i=1}^{n} p_{i} \bar{F}_{i}^{\alpha_{i}}(t)\right)\right) r_{1}(t) \\
& \quad \leq r_{1}(t)\left(\sum_{i=1}^{n} \alpha_{i} p_{i} \bar{F}_{i}^{\alpha_{i}}(t)\right)-\left(\left(\sum_{i=1}^{n} \alpha_{i} p_{i}\right)\left(\sum_{i=1}^{n} p_{i} \bar{F}_{i}^{\alpha_{i}}(t)\right)\right) r_{1}(t) .
\end{aligned}
$$


Thus

$$
\begin{aligned}
r(t, \bar{\alpha})-r_{1}(t) & \stackrel{\text { sign }}{=} r_{1}(t)\left[\alpha_{1} p_{1}\left(1-p_{1}\right) \bar{F}_{1}^{\alpha_{1}}(t)+\alpha_{2} p_{2}\left(1-p_{2}\right) \bar{F}_{2}^{\alpha_{2}}(t)+\cdots+\alpha_{n} p_{n}\left(1-p_{n}\right) \bar{F}_{n}^{\alpha_{n}}(t)\right. \\
& \left.-\alpha_{1} p_{1} \sum_{j \neq 1}^{n} p_{j} \bar{F}_{j}^{\alpha_{j}}(t)-\alpha_{2} p_{2} \sum_{j \neq 2}^{n} p_{j} \bar{F}_{j}^{\alpha_{j}}(t)-\cdots-\alpha_{n} p_{n} \sum_{j \neq n} p_{j} \bar{F}_{j}^{\alpha_{j}}(t)\right] \\
& =r_{1}(t)\left[\sum_{i=1}^{n} \alpha_{i} p_{i}\left(1-p_{i}\right) \bar{F}_{i}^{\alpha_{i}}(t)-\sum_{i=1}^{n} \alpha_{i} p_{i} \sum_{j \neq i}^{n} p_{j} \bar{F}_{j}^{\alpha_{j}}(t)\right] \\
& =r_{1}(t)\left[\sum_{i=1}^{n} \alpha_{i} p_{i}\left(\sum_{j \neq i}^{n} p_{j} \bar{F}_{i}^{\alpha_{i}}(t)-\sum_{j \neq i}^{n} p_{j} \bar{F}_{j}^{\alpha_{j}}(t)\right)\right] \\
& =r_{1}(t)\left[\sum_{i=1}^{n} \alpha_{i} p_{i}\left(\sum_{j \neq i}^{n} p_{j}\left(\bar{F}_{i}^{\alpha_{i}}(t)-\bar{F}_{j}^{\alpha_{j}}(t)\right)\right)\right] \\
& =r_{1}(t)\left[\sum_{i=2}^{n} p_{1} p_{i}\left(\bar{F}_{1}^{\alpha_{1}}(t)-\bar{F}_{i}^{\alpha_{i}}(t)\right)\left(\alpha_{1}-\alpha_{i}\right)\right. \\
& +\sum_{i=3}^{n} p_{2} p_{i}\left(\bar{F}_{2}^{\alpha_{2}}(t)-\bar{F}_{i}^{\alpha_{i}}(t)\right)\left(\alpha_{2}-\alpha_{i}\right)+\ldots \\
& \left.+p_{n-1} p_{n}\left(\bar{F}_{n-1}^{\alpha_{n-1}}(t)-\bar{F}_{n}^{\alpha_{n}}(t)\right)\left(\alpha_{n-1}-\alpha_{n}\right)\right] \\
& \leq r_{1}(t)\left[\sum_{i=2}^{n} p_{1} p_{i}\left(\bar{F}_{1}^{\alpha_{1}}(t)-\bar{F}_{1}^{\alpha_{i}}(t)\right)\left(\alpha_{1}-\alpha_{i}\right)\right. \\
& +\sum_{i=3}^{n} p_{2} p_{i}\left(\bar{F}_{2}^{\alpha_{2}}(t)-\bar{F}_{2}^{\alpha_{i}}(t)\right)\left(\alpha_{2}-\alpha_{i}\right)+\ldots \\
& \left.p_{n-1} p_{n}\left(\bar{F}_{n-1}^{\alpha_{n-1}}(t)-\bar{F}_{n-1}^{\alpha_{n}}(t)\right)\left(\alpha_{n-1}-\alpha_{n}\right)\right] \\
&
\end{aligned}
$$

As the hazard rate order implies the usual stochastic order, the assumption $F_{1} \leq_{h r} F_{2} \leq_{h r} \cdots \leq_{h r}$ $F_{n}$ yields $\bar{F}_{1}(t) \leq \bar{F}_{2}(t) \leq \cdots \leq \bar{F}_{n}(t)$. Thus, $\bar{F}_{i}^{\alpha_{j}}(t) \leq \bar{F}_{j}^{\alpha_{j}}(t)$ for $i \leq j, i, j=1,2, \ldots, n$, and then $\left(\bar{F}_{i}^{\alpha_{i}}(t)-\bar{F}_{j}^{\alpha_{j}}(t)\right) \leq\left(\bar{F}_{i}^{\alpha_{i}}(t)-\bar{F}_{i}^{\alpha_{j}}(t)\right)$ and last inequality holds.

Consider now the following two cases for $\alpha_{i}>0$ separately.

- Let $\alpha_{1} \geq \alpha_{2} \geq \cdots \geq \alpha_{n}$. In this case $\bar{F}_{i}^{\alpha_{i}}(t) \leq \bar{F}_{i}^{\alpha_{j}}(t)$ for $i \leq j, i, j=1,2, \ldots, n$ and then $\left(\bar{F}_{i}^{\alpha_{i}}(t)-\bar{F}_{i}^{\alpha_{j}}(t)\right) \leq 0$. Since $\left(\alpha_{i}-\alpha_{j}\right) \geq 0$ for $i \leq j, i, j=1,2, \ldots, n$, thus

$$
r(t, \bar{\alpha})-r_{1}(t) \leq 0 .
$$

- Let $\alpha_{1} \leq \alpha_{2} \leq \cdots \leq \alpha_{n}$. In this case $\bar{F}_{i}^{\alpha_{i}}(t) \geq \bar{F}_{i}^{\alpha_{j}}(t)$ for $i \leq j, i, j=1,2, \ldots, n$ and then $\left(\bar{F}_{i}^{\alpha_{i}}(t)-\bar{F}_{i}^{\alpha_{j}}(t)\right) \geq 0$. Since $\left(\alpha_{i}-\alpha_{j}\right) \leq 0$ for $i \leq j, i, j=1,2, \ldots, n$, thus

$$
r(t, \bar{\alpha})-r_{1}(t) \leq 0 .
$$


Thus, in general, $r(t, \bar{\alpha})-r_{1}(t) \leq 0$, which means that $F_{1} \leq_{h r} F(t, \bar{\alpha})$ for $\alpha_{i}>0, i=1, \ldots, n$. This complete the proof.

In the following, we study the closure property of the generalized finite $\alpha$-mixture. For proving the main result, we first, provide the following definition and lemma.

Definition 3.2. The hazard transform of the generalized finite $\alpha$-mixture is

$$
\eta_{\bar{\alpha}}(\mathbf{u})=-\frac{1}{\bar{\alpha}} \log \left(\sum_{i=1}^{n} p_{i} e^{-\alpha_{i} u_{i}}\right), \quad \alpha_{i} \in(-\infty, \infty),
$$

where $\mathbf{u}$ is a vector with elements $u_{i}, 0 \leq u_{i} \leq \infty$.

Thus, the hazard function $R(t, \bar{\alpha})=\int_{0}^{t} r(u, \bar{\alpha}) d u$ that corresponds to the lifetime described by the generalized finite $\alpha$-mixture is given by

$$
R(t, \bar{\alpha})=\eta_{\bar{\alpha}}(\mathbf{R}(t)) \equiv-\frac{1}{\bar{\alpha}} \log \left(\sum_{i=1}^{n} p_{i} e^{-\alpha_{i} R_{i}(t)}\right), \quad 0 \leq t<\infty,
$$

where $R_{i}(t)=\int_{0}^{t} r_{i}(u) d u, i=1, \ldots, n$ is the hazard function that corresponds to $\bar{F}_{i}(t)$ and $\mathbf{R}(t)$ is a vector.

Now we can prove the following lemma, which is an extension of Lemma A.1 of Asadi et al. (2019).

Lemma 3.3. The hazard transform $\eta_{\bar{\alpha}}(\mathbf{u})$ of the generalized finite $\alpha$-mixture is concave for $\alpha_{i}>0$ and convex for $\alpha_{i}<0$. That is, for $\alpha_{i}>0\left(\alpha_{i}<0\right)$

$$
\eta_{\bar{\alpha}}(\beta \mathbf{u}+(1-\beta) \mathbf{v}) \geq(\leq) \beta \eta_{\bar{\alpha}}(\mathbf{u})+(1-\beta) \eta_{\bar{\alpha}}(\mathbf{v})
$$

where $0 \leq \beta \leq 1$ and $0 \leq u_{i}, v_{i} \leq \infty$ for $i=1, . ., n$.

Proof. Using Holder's inequality we have (for $\left.\alpha_{i}>0\left(\alpha_{i}<0\right)\right)$ :

$$
\sum_{i=1}^{n} p_{i} e^{-\beta \alpha_{i} u_{i}} e^{-(1-\beta) \alpha_{i} v_{i}} \leq\left(\sum_{i=1}^{n} p_{i} e^{-\alpha_{i} u_{i}}\right)^{\beta}\left(\sum_{i=1}^{n} p_{i} e^{-\alpha_{i} v_{i}}\right)^{(1-\beta)} .
$$

Thus, the lemma follows from the definition of $\eta_{\bar{\alpha}}$.

In order to prove the corresponding closure theorem, we need also the following lemma from Barlow and Proschan (1975).

Lemma 3.4. If $h(u)$ is a concave (convex) function and is increasing in each argument and if $u(t)$ is concave (convex), then $g_{u}(t) \equiv h(u(t))$ is concave (convex).

Theorem 3.5. Let $\bar{F}(t, \bar{\alpha})$ be the generalized finite $\alpha$-mixture. Then:

(a) If each $\bar{F}_{i}(t)$ is DFR (IFR) then for $\alpha_{i}>0\left(\alpha_{i}<0\right) \bar{F}(t, \bar{\alpha})$ is DFR (IFR).

(b) If each $\bar{F}_{i}(t)$ is DFRA (IFRA) then for $\alpha_{i}>0\left(\alpha_{i}<0\right) \bar{F}(t, \bar{\alpha})$ is DFRA (IFRA). 
Proof. (a) Part (a) follows from Lemmas (3.3) and (3.4).

(b) As $\bar{F}_{i}(t)$ is DFRA (IFRA), and $\eta_{\bar{\alpha}}$ is increasing, the hazard function $R(t, \bar{\alpha})$ of the generalized finite $\alpha$-mixture satisfies

$$
\eta_{\bar{\alpha}}(\mathbf{R}(\beta t)) \geq(\leq) \eta_{\bar{\alpha}}(\beta \mathbf{R}(t)), \quad 0 \leq \beta \leq 1 .
$$

Choosing $\mathbf{v}=0$ in the result of Lemma 3.3, we obtain

$$
\eta_{\bar{\alpha}}(\beta \mathbf{R}(t)) \geq(\leq) \beta \eta_{\bar{\alpha}}(\mathbf{R}(t))
$$

Thus

$$
R(\beta t, \bar{\alpha}) \geq(\leq) \beta R(t, \bar{\alpha}),
$$

and hence, $\bar{F}(t, \bar{\alpha})$ is DFRA (IFRA).

The following example provides some applications of Theorems 3.1 and 3.5.

Example 3.6. Let $\bar{F}_{1}(t)=\exp (-0.8 t), t>0$ and $\bar{F}_{2}(t)=\exp (-0.4 t), t>0$. If we assume that $\left(p_{1}, p_{2}\right)=(0.7,0.3)$ and $\left(\alpha_{1}, \alpha_{2}\right)=(2,5)$, then clearly, $F_{1} \leq_{h r} F_{2}$. Since $\alpha_{i}>0, i=1,2$, then the conditions of Theorem 3.1 are satisfied. Thus, the $\alpha$-mixture hazard rate in this case is equal to

$$
r(t, \bar{\alpha})=\frac{1}{2.9} \times \frac{1.12 \exp (-1.6 t)+0.6 \exp (-2 t)}{\exp (-1.6 t)+\exp (-2 t)} .
$$

As an example for the Theorem 3.1, Figure 1 (a) depicts the plot of $r(t, \bar{\alpha})$ and hazard rate of the weakest sub-population $\left(r_{1}(t)=0.8\right)$. The plots show that, $F_{1} \leq_{h r} F(t, \bar{\alpha})$. On the other hand, one can see that $\bar{F}_{1}(t)$ and $\bar{F}_{2}(t)$ are DFR and, $\bar{F}(t, \bar{\alpha})$ is also DFR for $\alpha_{i}>0, i=1,2$. As an application of Theorem 3.5, set $\left(\alpha_{1}, \alpha_{2}\right)=(-2,-5)$. Similarly, we can see from Figure 1 (b) that, $F(t, \bar{\alpha}) \leq_{h r} F_{2}$ for $\alpha_{i}<0, i=1,2$. Also, $\bar{F}_{1}(t)$ and $\bar{F}_{2}(t)$ are IFR and, $\bar{F}(t, \bar{\alpha})$ is also IFR for $\alpha_{i}<0, i=1,2$.

\section{Conditional characteristics}

It is useful for further analysis in this section (see also the examples in the next section and Section 6, where we discuss stochastic comparisons using majorization technique) to rewrite some relationships (e.g., for the hazard rate functions) in a slightly different way. Then the relevant conditional characteristics will emerge naturally. For this, first consider a non-negative discrete random variable $\Lambda$ with probability mass $\pi\left(\lambda_{i}\right)=p_{i}$ at $\lambda=\lambda_{i}, i=1,2, \ldots, n$. Also, let $\bar{F}\left(t \mid \lambda_{i}\right)=$ $\bar{F}_{i}(t), f\left(t \mid \lambda_{i}\right)=f_{i}(t)$ and $r\left(t \mid \lambda_{i}\right)=r_{i}(t), i=1,2, \ldots, n$. Thus, the hazard rate that corresponds to the generalized finite $\alpha$-mixture can be given as follows:

$$
\begin{aligned}
r(t, \bar{\alpha}) & =\sum_{i=1}^{n} \frac{\alpha_{i}}{\bar{\alpha}} r_{i}(t) \frac{p_{i} \bar{F}_{i}^{\alpha_{i}}(t)}{\sum_{j=1}^{n} p_{j} \bar{F}_{j}^{\alpha_{j}}(t)} \\
& =\sum_{i=1}^{n} \frac{\alpha_{i}}{\bar{\alpha}} r\left(t \mid \lambda_{i}\right) \frac{\pi\left(\lambda_{i}\right) \bar{F}^{\alpha_{i}}\left(t \mid \lambda_{i}\right)}{\sum_{j=1}^{n} \pi\left(\lambda_{j}\right) \bar{F}^{\alpha_{j}}\left(t \mid \lambda_{j}\right)} \\
& =\sum_{i=1}^{n} \frac{\alpha_{i}}{\bar{\alpha}} r\left(t \mid \lambda_{i}\right) \pi_{\bar{\alpha}}\left(\lambda_{i} \mid t\right),
\end{aligned}
$$




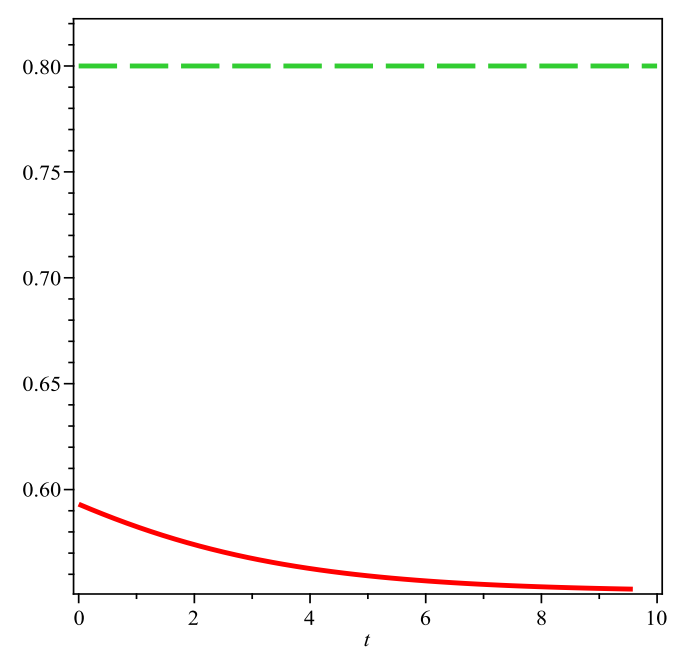

(a)

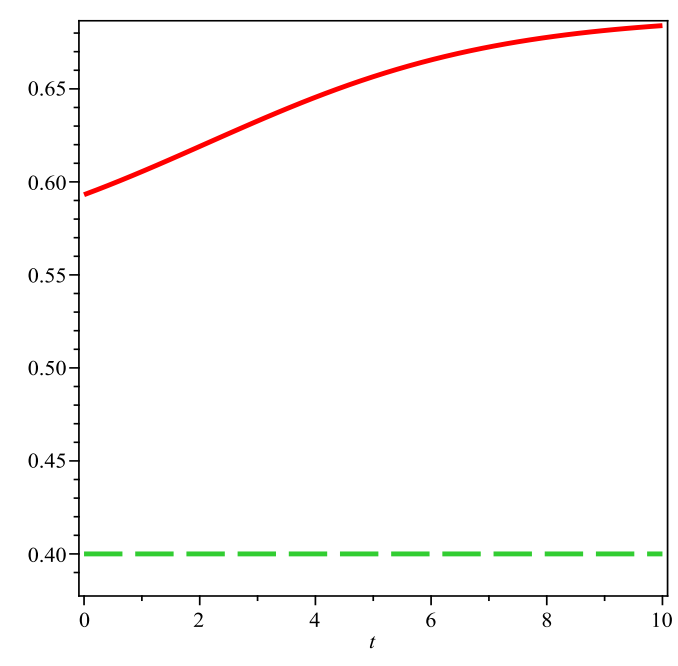

(b)

Figure 1: The plots of $r(t, \bar{\alpha})$ (solid) and the hazard rate of the weakest sub-population (dash dot) for $\alpha_{i}>0(\mathrm{a})$; the plots of $r(t, \bar{\alpha})$ (solid) and the hazard rate of the strongest sub-population (dash dot) for $\alpha_{i}<0(\mathrm{~b})$.

where

$$
\pi_{\bar{\alpha}}\left(\lambda_{i} \mid t\right)=\frac{\pi\left(\lambda_{i}\right) \bar{F}^{\alpha_{i}}\left(t \mid \lambda_{i}\right)}{\sum_{j=1}^{n} \pi\left(\lambda_{j}\right) \bar{F}^{\alpha_{j}}\left(t \mid \lambda_{j}\right)},
$$

is the conditional probability mass at $\lambda=\lambda_{i}, i=1,2, \ldots, n$ and $\bar{\alpha}=\sum_{i=1}^{n} \alpha_{i} \pi\left(\lambda_{i}\right)$ is the weighted average of $\alpha_{i}, i=1,2, \ldots, n$, with weights $\pi\left(\lambda_{i}\right)$.

Denote by $E_{\bar{\alpha}}(\Lambda \mid t)$ the conditional expectation of $\Lambda$, that is,

$$
E_{\bar{\alpha}}(\Lambda \mid t)=\sum_{i=1}^{n} \lambda_{i} \pi_{\bar{\alpha}}\left(\lambda_{i} \mid t\right)
$$

and by $\phi_{\bar{\alpha}}(t)$ the derivative of $E_{\bar{\alpha}}(\Lambda \mid t)$ with respect to $t$, i.e.,

$$
\phi_{\bar{\alpha}}(t)=\frac{\partial}{\partial t} E_{\bar{\alpha}}(\Lambda \mid t)=\sum_{i=1}^{n} \lambda_{i} \frac{\partial}{\partial t} \pi_{\bar{\alpha}}\left(\lambda_{i} \mid t\right),
$$


where

$$
\begin{aligned}
\frac{\partial}{\partial t} \pi_{\bar{\alpha}}\left(\lambda_{i} \mid t\right) & =\frac{\left[-\pi\left(\lambda_{i}\right) \alpha_{i} f\left(t \mid \lambda_{i}\right) \bar{F}^{\alpha_{i}-1}\left(t \mid \lambda_{i}\right)\right]\left[\sum_{j=1}^{n} \pi\left(\lambda_{j}\right) \bar{F}^{\alpha_{j}}\left(t \mid \lambda_{j}\right)\right]}{\left[\sum_{j=1}^{n} \pi\left(\lambda_{j}\right) \bar{F}^{\alpha_{j}}\left(t \mid \lambda_{j}\right)\right]^{2}} \\
& +\frac{\left[\sum_{j=1}^{n} \pi\left(\lambda_{j}\right) \alpha_{j} f\left(t \mid \lambda_{j}\right) \bar{F}^{\alpha_{j}-1}\left(t \mid \lambda_{j}\right)\right]\left[\pi\left(\lambda_{i}\right) \bar{F}^{\alpha_{i}}\left(t \mid \lambda_{i}\right)\right]}{\left[\sum_{j=1}^{n} \pi\left(\lambda_{j}\right) \bar{F}^{\alpha_{j}}\left(t \mid \lambda_{j}\right)\right]^{2}} \\
& =\frac{\left[\pi\left(\lambda_{i}\right) \bar{F}^{\alpha_{i}}\left(t \mid \lambda_{i}\right)\right]\left[\sum_{j=1}^{n} \alpha_{j} r\left(t \mid \lambda_{j}\right) \pi\left(\lambda_{j}\right) \bar{F}^{\alpha_{j}}\left(t \mid \lambda_{j}\right)\right]}{\left[\sum_{j=1}^{n} \pi\left(\lambda_{j}\right) \bar{F}^{\alpha_{j}}\left(t \mid \lambda_{j}\right)\right]^{2}}-\frac{\alpha_{i} r\left(t \mid \lambda_{i}\right) \pi\left(\lambda_{i}\right) \bar{F}^{\alpha_{i}}\left(t \mid \lambda_{i}\right)}{\sum_{j=1}^{n} \pi\left(\lambda_{j}\right) \bar{F}^{\alpha_{j}}\left(t \mid \lambda_{j}\right)} \\
& =\pi_{\bar{\alpha}}\left(\lambda_{i} \mid t\right) \bar{\alpha} r(t, \bar{\alpha})-\alpha_{i} r\left(t \mid \lambda_{i}\right) \pi_{\bar{\alpha}}\left(\lambda_{i} \mid t\right) .
\end{aligned}
$$

Thus,

$$
\begin{aligned}
\phi_{\bar{\alpha}}(t) & =\bar{\alpha}\left[r(t, \bar{\alpha}) E_{\bar{\alpha}}(\Lambda \mid t)-\sum_{i=1}^{n} \frac{\alpha_{i}}{\bar{\alpha}} \lambda_{i} r\left(t \mid \lambda_{i}\right) \pi_{\bar{\alpha}}\left(\lambda_{i} \mid t\right)\right] \\
& =\bar{\alpha}\left[E_{\bar{\alpha}}\left(r^{*}(t \mid \Lambda) \mid t\right) E_{\bar{\alpha}}(\Lambda \mid t)-E_{\bar{\alpha}}\left(\Lambda r^{*}(t \mid \Lambda) \mid t\right)\right] \\
& =-\bar{\alpha} \operatorname{Cov}_{\bar{\alpha}}\left(\left(\Lambda, r^{*}(t \mid \Lambda)\right) \mid t\right),
\end{aligned}
$$

where $r^{*}\left(t \mid \lambda_{i}\right)=\frac{\alpha_{i}}{\bar{\alpha}} r\left(t \mid \lambda_{i}\right)$ for $i=1,2, \ldots, n$. Now, we can formulate the following results:

- If $r(t \mid \lambda)$ is increasing in $\lambda$ and $\alpha_{1} \leq \alpha_{2} \leq \cdots \leq \alpha_{n}\left(\alpha_{1} \geq \alpha_{2} \geq \cdots \geq \alpha_{n}\right)$ for $\alpha_{i} \geq 0\left(\alpha_{i} \leq 0\right)$, $i=1,2, \ldots, n$, then $\operatorname{Cov}_{\bar{\alpha}}\left(\left(\Lambda, r^{*}(t \mid \Lambda)\right) \mid t\right)>0$, which, in turn, implies that for $\alpha_{i} \geq 0\left(\alpha_{i} \leq 0\right)$, $i=1,2, \ldots, n, \phi_{\bar{\alpha}}(t)<0(>0)$. This means that $E_{\bar{\alpha}}(\Lambda \mid t)$ is decreasing (increasing) in $t$.

- If $r(t \mid \lambda)$ is decreasing in $\lambda$ and $\alpha_{1} \geq \alpha_{2} \geq \cdots \geq \alpha_{n}\left(\alpha_{1} \leq \alpha_{2} \leq \cdots \leq \alpha_{n}\right)$ for $\alpha_{i} \geq 0\left(\alpha_{i} \leq 0\right)$, $i=1,2, \ldots, n$, then $\operatorname{Cov}_{\bar{\alpha}}\left(\left(\Lambda, r^{*}(t \mid \Lambda)\right) \mid t\right)<0$, which, in turn, implies that for $\alpha_{i} \geq 0\left(\alpha_{i} \leq 0\right)$, $i=1,2, \ldots, n, \phi_{\bar{\alpha}}(t)>0(<0)$. This means that $E_{\bar{\alpha}}(\Lambda \mid t)$ is increasing (decreasing) in $t$.

\section{Special cases: additive and multiplicative models}

It is well-known that the additive hazard model has the following form:

$$
r\left(t \mid \lambda_{i}\right)=r(t)+\lambda_{i}, \quad i=1,2, \ldots, n,
$$

where $r(t)$ is the baseline hazard rate. In this case, the hazard rate that corresponds to the generalized finite $\alpha$-mixture, $r(t, \bar{\alpha})$, from (8) can be given as follows:

$$
\begin{aligned}
r(t, \bar{\alpha}) & \left.=\sum_{i=1}^{n} \frac{\alpha_{i}}{\bar{\alpha}}\left(r(t)+\lambda_{i}\right)\right) \pi_{\bar{\alpha}}\left(\lambda_{i} \mid t\right) \\
& =r(t) \sum_{i=1}^{n} \frac{\alpha_{i}}{\bar{\alpha}} \pi_{\bar{\alpha}}\left(\lambda_{i} \mid t\right)+\sum_{i=1}^{n} \frac{\alpha_{i}}{\bar{\alpha}} \lambda_{i} \pi_{\bar{\alpha}}\left(\lambda_{i} \mid t\right) \\
& =r(t) \frac{\bar{\alpha}^{*}}{\bar{\alpha}}+E_{\bar{\alpha}}\left(\Lambda^{*} \mid t\right),
\end{aligned}
$$


where $\lambda_{i}^{*}=\frac{\alpha_{i}}{\bar{\alpha}} \lambda_{i}, i=1,2, \ldots, n$ and $\bar{\alpha}^{*}=\sum_{i=1}^{n} \alpha_{i} \pi_{\bar{\alpha}}\left(\lambda_{i} \mid t\right)$ is the weighted average of $\alpha_{i}, i=$ $1,2, \ldots, n$, with weights $\pi_{\bar{\alpha}}\left(\lambda_{i} \mid t\right)$.

For the multiplicative hazard model, we have:

$$
r\left(t \mid \lambda_{i}\right)=\lambda_{i} r(t), \quad i=1,2, \ldots, n .
$$

Hence, the hazard rate that corresponds to the generalized finite $\alpha$-mixture, $r(t, \bar{\alpha})$, is

$$
\begin{aligned}
r(t, \bar{\alpha}) & =\sum_{i=1}^{n} \frac{\alpha_{i}}{\bar{\alpha}} \lambda_{i} r(t) \pi_{\bar{\alpha}}\left(\lambda_{i} \mid t\right) \\
& =r(t) E_{\bar{\alpha}}\left(\Lambda^{*} \mid t\right) .
\end{aligned}
$$

Thus

$$
r^{\prime}(t, \bar{\alpha})=r^{\prime}(t) E_{\bar{\alpha}}\left(\Lambda^{*} \mid t\right)+r(t) \frac{\partial}{\partial t} E_{\bar{\alpha}}\left(\Lambda^{*} \mid t\right) .
$$

From representation (13), the generalized finite $\alpha$-mixture will be IFR (DFR) for $\alpha_{i}>0\left(\alpha_{i}<0\right)$, $i=1,2, \ldots, n$, if and only if for all $t \in(0, \infty)$

$$
\frac{r^{\prime}(t)}{r(t)} \geq(\leq)-\frac{\frac{\partial}{\partial t} E_{\bar{\alpha}}\left(\Lambda^{*} \mid t\right)}{E_{\bar{\alpha}}\left(\Lambda^{*} \mid t\right)} .
$$

The following theorem describes the limiting behavior of hazard rates for the multiplicative model in the generalized $\alpha$-mixture model for two components. The result is given for positive values of $\alpha_{i}, i=1,2$. The case of negative values can be described in a similar manner.

Theorem 4.1. Let $r_{1}(t)=\lambda_{1} r(t), r_{2}(t)=\lambda_{2} r(t)$ where $\lambda_{1} \leq \lambda_{2}$ and $r(t) \longrightarrow \infty$ as $t \longrightarrow \infty$ in the multiplicative model for the generalized finite $\alpha$-mixture of two SFs with $\alpha_{i}>0$. If $\alpha_{1} \leq$ c $\alpha_{2}$, where $c=\frac{\lambda_{2}}{\lambda_{1}} \geq 1$ and $\bar{\alpha}=p_{1} \alpha_{1}+p_{2} \alpha_{2}$, then

(a)

$$
r(t, \bar{\alpha})=\frac{\alpha_{1}}{\bar{\alpha}} r_{1}(t)(1+o(1)) \quad \text { as } t \longrightarrow \infty .
$$

(b)

if and only if

$$
r(t, \bar{\alpha})-\frac{\alpha_{1}}{\bar{\alpha}} r_{1}(t) \longrightarrow 0 \text { as } t \longrightarrow \infty
$$

$$
r(t) \exp \left\{-\left(\alpha_{2} \lambda_{2}-\alpha_{1} \lambda_{1}\right) \int_{0}^{t} r(u) d u\right\} \rightarrow 0 \text { as } t \rightarrow \infty
$$

Proof. From assumptions of the theorem, we have $r_{2}(t)=c r_{1}(t)$, where $c=\frac{\lambda_{2}}{\lambda_{1}} \geq 1$. This implies that $\bar{F}_{2}(t)=\bar{F}_{1}^{c}(t)$ and $f_{2}(t)=c f_{1}(t) \bar{F}_{1}^{c-1}(t)$. Thus, the ratio of the two densities and the two survival functions as $t \longrightarrow \infty$, is respectively,

$$
\begin{aligned}
& \frac{f_{1}(t)}{f_{2}(t)}=\frac{\bar{F}_{1}^{1-c}(t)}{c} \uparrow \infty \text { if } c>1 \text { and }=1 \text { if } c=1 \\
& \frac{\bar{F}_{1}(t)}{\bar{F}_{2}(t)}=\bar{F}_{1}^{1-c}(t) \uparrow \infty \text { if } c>1 \text { and }=1 \text { if } c=1 .
\end{aligned}
$$


We have

$$
\begin{aligned}
\frac{r(t, \bar{\alpha})}{r_{1}(t)} & =\frac{1}{\bar{\alpha}} \times \frac{\bar{F}_{1}^{\alpha_{1}-1}(t)\left[\alpha_{1} p_{1}+c \alpha_{2} p_{2} \bar{F}_{1}^{c \alpha_{2}-\alpha_{1}}(t)\right] \times \bar{F}_{1}(t)}{\bar{F}_{1}^{\alpha_{1}}(t)\left[p_{1}+p_{2} \bar{F}_{1}^{c \alpha_{2}-\alpha_{1}}(t)\right]} \\
& =\frac{1}{\bar{\alpha}} \times \frac{\left[\alpha_{1} p_{1} \bar{F}_{1}^{\alpha_{1}-c \alpha_{2}}(t)+c \alpha_{2} p_{2}\right] \bar{F}_{1}^{c \alpha_{2}-\alpha_{1}}(t)}{\left[p_{1} \bar{F}_{1}^{\alpha_{1}-c \alpha_{2}}(t)+p_{2}\right] \bar{F}_{1}^{c \alpha_{2}-\alpha_{1}}(t)} \\
& =\frac{1}{\bar{\alpha}} \times\left(\alpha_{1}+\frac{p_{2}\left(c \alpha_{2}-\alpha_{1}\right)}{p_{1} \bar{F}_{1}^{\alpha_{1}-c \alpha_{2}}(t)+p_{2}}\right) .
\end{aligned}
$$

When $t \rightarrow \infty, \bar{F}_{1}(t) \rightarrow 0$ and thus, we get relationship (14). To prove (b), note that

$$
r(t, \bar{\alpha})-\frac{\alpha_{1}}{\bar{\alpha}} r_{1}(t)=\frac{1}{\bar{\alpha}} \times r_{1}(t) \bar{F}_{1}^{c \alpha_{2}-\alpha_{1}}(t) \frac{p_{2}\left(c \alpha_{2}-\alpha_{1}\right)}{p_{1}+p_{2} \bar{F}_{1}^{c \alpha_{2}-\alpha_{1}}(t)} .
$$

As

$$
r_{1}(t) \bar{F}_{1}^{c \alpha_{2}-\alpha_{1}}(t)=\lambda_{1} r(t) \exp \left\{-\left(\alpha_{2} \lambda_{2}-\alpha_{1} \lambda_{1}\right) \int_{0}^{t} r(u) d u\right\}
$$

from (16), we get

$$
r_{1}(t) \bar{F}_{1}^{c \alpha_{2}-\alpha_{1}}(t) \longrightarrow 0 \text { as } t \longrightarrow \infty,
$$

Hence, from (17), we obtain the result in (15). This completes the proof.

Remark 4.2. In Theorem 4.1, since $\lambda_{1} \leq \lambda_{2}$, we have $F_{1} \geq_{h r} F_{2}$, and from the results of section 3, before the Theorem 3.1, we have $\frac{\alpha_{1}}{\bar{\alpha}} r_{1}(t) \leq r(t, \bar{\alpha})$. The results of Theorem 4.1 show the asymptotic behavior of $r(t, \bar{\alpha})$ in multiplicative model. In particular, if $\alpha_{i}=\alpha>0\left(\alpha_{i}=\alpha<0\right), i=1,2$, the hazard rate of the generalized finite $\alpha$-mixture in multiplicative model tends to the hazard rate of the strongest (weakest) sub-population as $t \rightarrow \infty$.

\section{Stochastic comparisons for different baseline distributions}

Our main emphasis in the rest of the paper will be on stochastic comparisons obtained by using majorization technique. However, as a prelude to that, in this section, we discuss meaningful stochastic comparisons for two generalized finite $\alpha$-mixtures in the more conventional for existing literature way.

Theorem 5.1. Let $F_{p}(t, \bar{\alpha})$ and $G_{p}(t, \bar{\alpha})$ be two n-component generalized finite $\alpha$-mixture models with common mixing probabilities $\left(p_{1}, \ldots, p_{n}\right)$. Assume that for $\alpha_{i}>0\left(\alpha_{i}<0\right), F_{i} \leq_{s t} G_{i}$ for $i=1, \ldots, n$. Then

$$
F_{p}(t, \bar{\alpha}) \leq_{s t} G_{p}(t, \bar{\alpha}) .
$$

Proof. Let $\alpha_{i}>0\left(\alpha_{i}<0\right)$. From $F_{i} \leq_{s t} G_{i}$ for $i=1, \ldots, n$, we have $\bar{F}_{i}(t) \leq \bar{G}_{i}(t)$ for any $t$, $i=1, \ldots, n$. Since $\alpha_{i}>0\left(\alpha_{i}<0\right), \bar{F}_{i}^{\alpha_{i}}(t) \leq(\geq) \bar{G}_{i}^{\alpha_{i}}(t)$ for $i=1, \ldots, n$. Thus,

$$
\sum_{i=1}^{n} p_{i} \bar{F}_{i}^{\alpha_{i}} \leq(\geq) \sum_{i=1}^{n} p_{i} \bar{G}_{i}^{\alpha_{i}} .
$$


Consequently from $\sum_{i=1}^{n} p_{i} \alpha_{i}>0\left(\sum_{i=1}^{n} p_{i} \alpha_{i}<0\right)$

$$
\left[\sum_{i=1}^{n} p_{i} \bar{F}_{i}^{\alpha_{i}}\right]^{\sum_{i=1}^{n} p_{i} \alpha_{i}} \leq\left[\sum_{i=1}^{n} p_{i} \bar{G}_{i}^{\alpha_{i}}\right]^{\sum_{i=1}^{n} p_{i} \alpha_{i}} .
$$

This means for $\alpha_{i}>0\left(\alpha_{i}<0\right)$

$$
F_{p}(t, \bar{\alpha}) \leq_{s t} G_{p}(t, \bar{\alpha}) .
$$

Theorem 5.2. Let $F_{p}(t, \bar{\alpha})$ and $G_{p}(t, \bar{\alpha})$ be two $n$-component generalized finite $\alpha$-mixture models with common mixed proportions $\left(p_{1}, \ldots, p_{n}\right)$. Suppose that for $\alpha_{i}>0\left(\alpha_{i}<0\right)$

(i) $\frac{\alpha_{1}}{\bar{\alpha}} r_{F_{1}}(t) \leq \cdots \leq \frac{\alpha_{n}}{\bar{\alpha}} r_{F_{n}}(t)$ or $\frac{\alpha_{1}}{\bar{\alpha}} r_{G_{1}}(t) \leq \cdots \leq \frac{\alpha_{n}}{\bar{\alpha}} r_{G_{n}}(t)$;

(ii) $\frac{\bar{F}_{i}(t)}{\bar{G}_{i}(t)}$ is increasing (decreasing) in $i \in\{1,2, \ldots, n\}$;

(iii) $F_{i} \leq_{h r} G_{i}$ for all $i \in\{1,2, \ldots, n\}$;

(iv) $\alpha_{1} \geq \ldots \geq \alpha_{n}$

Then

$$
F_{p}(t, \bar{\alpha}) \leq_{h r} G_{p}(t, \bar{\alpha}) .
$$

Proof. Let $\alpha_{i}>0$. From (4), the expressions for the failure rate functions that correspond to $F_{p}(t, \bar{\alpha})$ and $G_{p}(t, \bar{\alpha})$ can be written as

$$
r_{F}(t, \bar{\alpha})=\sum_{i=1}^{n} \frac{\alpha_{i}}{\bar{\alpha}} r_{F_{i}}(t) p_{\alpha_{i}}(t)
$$

and

$$
r_{G}(t, \bar{\alpha})=\sum_{i=1}^{n} \frac{\alpha_{i}}{\bar{\alpha}} r_{G_{i}}(t) q_{\alpha_{i}}(t)
$$

respectively, where $q_{\alpha_{i}}(t)=\frac{p_{i} \bar{G}_{i}^{\alpha_{i}}(t)}{\sum_{j=1}^{n} p_{j} \bar{G}_{j}^{\alpha_{j}}(t)}$ for $i=1, \ldots, n$. To prove $F_{p}(t, \bar{\alpha}) \leq_{h r} G_{p}(t, \bar{\alpha})$ for $\alpha_{i}>0$, we need to show that $\psi(t)=r_{F}(t, \bar{\alpha})-r_{G}(t, \bar{\alpha})$ is non-negative for all $t \geq 0$. Note that,

$$
\begin{aligned}
\psi(t) & =\sum_{i=1}^{n} \frac{\alpha_{i}}{\bar{\alpha}} r_{F_{i}}(t) p_{\alpha_{i}}(t)-\sum_{i=1}^{n} \frac{\alpha_{i}}{\bar{\alpha}} r_{G_{i}}(t) q_{\alpha_{i}}(t) \\
& \geq \sum_{i=1}^{n} \frac{\alpha_{i}}{\bar{\alpha}} r_{G_{i}}(t) p_{\alpha_{i}}(t)-\sum_{i=1}^{n} \frac{\alpha_{i}}{\bar{\alpha}} r_{G_{i}}(t) q_{\alpha_{i}}(t) \equiv \xi(t),
\end{aligned}
$$

where the inequality follows from condition ( $i i i)$. Thus, it suffices to show that $\xi(t)$ is non-negative for all $t \geq 0$. Consider two non-negative discrete random variables $W$ and $V$ on a sample space $\{1, \ldots, n\}$ with probability mass functions $q_{\alpha_{i}}(t)$ and $p_{\alpha_{i}}(t), i=1, \ldots, n$, respectively. Thus, $\xi(t)$ can be written as

$$
\xi(t)=E[\phi(V)]-E[\phi(W)]
$$


where $\phi(i)=\frac{\alpha_{i}}{\bar{\alpha}} r_{G_{i}}(),. i=1, \ldots, n$. To prove that (18) is non-negative, it is sufficient to show that $\phi(i)$ is increasing in $i$ and $W \leq_{s t} V$. Based on condition $(i)$, we have $\frac{\alpha_{1}}{\bar{\alpha}} r_{G_{1}}(t) \leq \cdots \leq \frac{\alpha_{n}}{\bar{\alpha}} r_{G_{n}}(t)$ for all $t \geq 0$. Thus, $\phi(i)$ is increasing in $i$. On the other hand, one can see that

$$
\frac{p_{\alpha_{i}}(t)}{q_{\alpha_{i}}(t)} \propto\left(\frac{\bar{F}_{i}(t)}{\bar{G}_{i}(t)}\right)^{\alpha_{i}} i \in\{1, \ldots, n\} .
$$

The condition (iii), i.e., $F_{i} \leq_{h r} G_{i}$ implies $\bar{F}_{i}(t) \leq \bar{G}_{i}(t), i \in\{1,2, \ldots, n\}$. Thus, $0 \leq \frac{\bar{F}_{i}(t)}{\bar{G}_{i}(t)} \leq 1$ for all $i \in\{1,2, \ldots, n\}$. Hence, conditions $(i i)$ and $(i v)$ imply that $\frac{p_{\alpha_{i}}(t)}{q_{\alpha_{i}}(t)}$ is increasing in $i \in\{1, \ldots, n\}$, which means that $W \leq_{l r} V$, which in turn implies $W \leq_{s t} V$. Thus, $\xi(t)$ is non-negative and for $\alpha_{i}>0, F_{p}(t, \bar{\alpha}) \leq_{h r} G_{p}(t, \bar{\alpha})$. If, we assume $\frac{\alpha_{1}}{\bar{\alpha}} r_{F_{1}}(t) \leq \cdots \leq \frac{\alpha_{n}}{\bar{\alpha}} r_{F_{n}}(t)$, the proof is similar. The case $\alpha_{i}<0$ can be also established in the same way.

\section{Stochastic comparisons using majorization concept}

In this section, we provide the main comparison results of the paper with detailed analysis, examples and counterexamples. Specifically, we compare two generalized finite $\alpha$-mixture in the sense of the usual stochastic order and in the sense of the hazard rate order when the vector of parameters of the first mixture majorizes the second one (see the relevant conditional characteristics discussed in Sections 3 and 4). However, first, we must recall some definitions and supplementary results to be used in what follows.

Definition 6.1. (Marshall et al. (2011), p. 8). Consider two vectors $\boldsymbol{x}=\left(x_{1}, \ldots, x_{n}\right)$ and $\boldsymbol{y}=\left(y_{1}, \ldots, y_{n}\right)$. Let $x_{(1)} \leq \cdots \leq x_{(n)}$ and $y_{(1)} \leq \cdots \leq y_{(n)}$ be increasing arrangements of their components, respectively.

(i) If $\sum_{j=1}^{i} x_{(j)} \leq \sum_{j=1}^{i} y_{(j)}$ for $i=1, \ldots, n-1$, and $\sum_{j=1}^{i} x_{(j)}=\sum_{j=1}^{i} y_{(j)}$, then $\boldsymbol{x}$ is said to majorize $\boldsymbol{y}$ and denoted by $\boldsymbol{x} \succeq \boldsymbol{m}$.

(ii) If $\sum_{j=1}^{i} x_{(j)} \leq \sum_{j=1}^{i} y_{(j)}$ for $i=1, \ldots, n$, then $\boldsymbol{x}$ is said to weakly supermajorize $\boldsymbol{y}$, and denoted by $\boldsymbol{x} \succeq \boldsymbol{y}$.

(iii) If $\sum_{j=i}^{n} x_{(j)} \geq \sum_{j=i}^{n} y_{(j)}$ for $i=1, \ldots, n$, then we say that $\boldsymbol{x}$ weakly submajorize $\boldsymbol{y}$, denoted by $\boldsymbol{x} \succeq_{w} \boldsymbol{y}$.

From the foregoing definition, clearly, the majorization order implies both weak submajorization and supermajorization orders. Order-preserving functions have been widely used in the literature to obtain some inequalities that arise from majorization. A Schur-convex function is a function that preserves the ordering of majorization (Marshall et al., 2011).

Definition 6.2. (Marshall et al. (2011), p. 80). A real-valued function $\phi$ defined on a set $\mathbb{A} \subseteq \mathbb{R}^{n}$ is said to be Schur-convex (Schur-concave) on $\mathbb{A}$ if $\boldsymbol{x} \succeq \boldsymbol{m}$ implies $\phi(\boldsymbol{x}) \geq(\leq) \phi(\boldsymbol{y})$ for any $\boldsymbol{x}, \boldsymbol{y} \in \mathbb{A}$.

Some conditions for characterization of Schur-convex (Schur-concave) functions are provided in the next lemma. 
Lemma 6.3. (Marshall et al. (2011), p. 84). Let $I \subseteq \mathbb{R}$ be an open interval and let $\phi: I \rightarrow \mathbb{R}$ be a real-valued, continuously differentiable function. Then, $\phi$ is Schur-convex (Schur-concave) on $I^{n}$ if and only if

(i) $\phi$ is symmetric on $I^{n}$, and

(ii) for all $i \neq j$ and all $\boldsymbol{x} \in I^{n}$,

$$
\left(x_{i}-x_{j}\right)\left(\frac{\partial \phi}{\partial x_{i}}(\boldsymbol{x})-\frac{\partial \phi}{\partial x_{j}}(\boldsymbol{x})\right) \geq 0(\leq 0)
$$

where the partial derivative of $\phi$ with respect to its $i$-th argument denoted by $\frac{\partial \phi}{\partial x_{i}}$.

The next lemma provides some conditions under which the weak supermajorization and the weak submajorization orders are preserved.

Lemma 6.4. (Marshall et al. (2011), p. 87). Consider the real-valued function $\phi$, defined on a set $\mathbb{A} \subseteq \mathbb{R}^{n}$. Then,

(i) $\boldsymbol{x} \succeq_{w} \boldsymbol{y}$ implies $\phi(\boldsymbol{x}) \geq \phi(\boldsymbol{y})$ if and only if $\phi$ is increasing and Schur-convex on $\mathbb{A}$;

(ii) $\boldsymbol{x} \succeq \boldsymbol{w}$ implies $\phi(\boldsymbol{x}) \geq \phi(\boldsymbol{y})$ if and only if $\phi$ is decreasing and Schur-convex on $\mathbb{A}$.

We will also need the following notation

$$
\mathcal{U}_{n}=\left\{(\boldsymbol{x}, \boldsymbol{y}): x_{i}, y_{i} \geq 0 \quad\left(x_{i}-x_{j}\right)\left(y_{i}-y_{j}\right) \leq 0, \quad i, j=1, \ldots, n\right\}
$$

\subsection{Usual stochastic order}

Theorem 6.5. Let

$$
\left.\bar{F}_{W_{n}(\boldsymbol{p}, \boldsymbol{\lambda}}\right)\left(t, \bar{\alpha}_{p}\right)=\left[\sum_{i=1}^{n} p_{i} \bar{F}^{\alpha_{i}}\left(t \mid \lambda_{i}\right)\right]^{1 / \bar{\alpha}_{p}}
$$

and

$$
\bar{F}_{W_{n}(\boldsymbol{p}, \boldsymbol{\gamma})}\left(t, \bar{\alpha}_{p}\right)=\left[\sum_{i=1}^{n} p_{i} \bar{F}^{\alpha_{i}}\left(t \mid \gamma_{i}\right)\right]^{1 / \bar{\alpha}_{p}},
$$

be two $n$-component generalized finite $\alpha$-mixtures with common mixing proportions $p_{1}, \ldots, p_{n}$ and parameters $\lambda_{1}, \ldots, \lambda_{n}$ and $\gamma_{1}, \ldots, \gamma_{n}$, respectively, where $\bar{\alpha}_{p}=\sum_{i=1}^{n} p_{i} \alpha_{i}$. Suppose that for $(\boldsymbol{p}, \boldsymbol{\lambda}) \in$ $\mathcal{U}_{n}$ and $(\boldsymbol{p}, \gamma) \in \mathcal{U}_{n}:$

(i) $\bar{F}(t \mid \lambda)$ is decreasing and convex in $\lambda>0$ for all $t \geq 0$;

(ii) $\alpha_{1} \leq \ldots, \leq \alpha_{n}$;

(iii) $\lambda \stackrel{w}{\succeq} \gamma$;

(iv) $\alpha_{i} p_{i} \geq \alpha_{j} p_{j}$ for all $i \leq j, i, j=1,2, \ldots, n$. 
Then for $\alpha_{i} \geq 1$ :

$$
\bar{F}_{W_{n}(\boldsymbol{p}, \boldsymbol{\lambda})}\left(t, \bar{\alpha}_{p}\right) \geq \bar{F}_{W_{n}(\boldsymbol{p}, \boldsymbol{\gamma})}\left(t, \bar{\alpha}_{p}\right) .
$$

Proof. Without loss of generality, suppose that $p_{1} \geq p_{2} \geq \cdots \geq p_{n}$. From assumption $(\boldsymbol{p}, \boldsymbol{\lambda}) \in \mathcal{U}_{n}$ and $(\boldsymbol{p}, \gamma) \in \mathcal{U}_{n}$, we have $0<\lambda_{1} \leq \lambda_{2} \leq \cdots \leq \lambda_{n}$ and $0<\gamma_{1} \leq \gamma_{2} \leq \cdots \leq \gamma_{n}$. Note that, by considering $\phi(\boldsymbol{\lambda})=\bar{F}_{W_{n}(\boldsymbol{p}, \boldsymbol{\lambda})}\left(t, \bar{\alpha}_{p}\right)=\left[\sum_{i=1}^{n} p_{i} \bar{F}^{\alpha_{i}}\left(t \mid \lambda_{i}\right)\right]^{1 / \bar{\alpha}_{p}}$, we have

$$
\frac{\partial \phi(\boldsymbol{\lambda})}{\partial \lambda_{i}}=p_{i} \frac{\alpha_{i}}{\bar{\alpha}_{p}}\left(\frac{\partial \bar{F}\left(t \mid \lambda_{i}\right)}{\partial \lambda_{i}}\right) \bar{F}^{\alpha_{i}-1}\left(t \mid \lambda_{i}\right)\left[\sum_{i=1}^{n} p_{i} \bar{F}^{\alpha_{i}}\left(t \mid \lambda_{i}\right)\right]^{\frac{1}{\bar{\alpha}_{p}}-1} \leq 0,
$$

as from condition $(i), \bar{F}\left(t \mid \lambda_{i}\right)$ is decreasing in $\lambda_{i}$. In addition, from Lemma 6.3 and for any pair $(i, j), 1 \leq i<j \leq n$,

$$
\begin{aligned}
\frac{\partial \phi(\boldsymbol{\lambda})}{\partial \lambda_{i}}-\frac{\partial \phi(\boldsymbol{\lambda})}{\partial \lambda_{j}} & =p_{i} \frac{\alpha_{i}}{\bar{\alpha}_{p}}\left(\frac{\partial \bar{F}\left(t \mid \lambda_{i}\right)}{\partial \lambda_{i}}\right) \bar{F}^{\alpha_{i}-1}\left(t \mid \lambda_{i}\right)\left[\sum_{i=1}^{n} p_{i} \bar{F}^{\alpha_{i}}\left(t \mid \lambda_{i}\right)\right]^{\frac{1}{\bar{\alpha}_{p}}-1} \\
& -p_{j} \frac{\alpha_{j}}{\bar{\alpha}_{p}}\left(\frac{\partial \bar{F}\left(t \mid \lambda_{j}\right)}{\partial \lambda_{j}}\right) \bar{F}^{\alpha_{j}-1}\left(t \mid \lambda_{j}\right)\left[\sum_{i=1}^{n} p_{i} \bar{F}^{\alpha_{i}}\left(t \mid \lambda_{i}\right)\right]^{\frac{1}{\bar{\alpha}_{p}}-1} .
\end{aligned}
$$

This implies that

$$
\begin{aligned}
& \frac{\partial \phi(\boldsymbol{\lambda})}{\partial \lambda_{i}}-\frac{\partial \phi(\boldsymbol{\lambda})}{\partial \lambda_{j}} \\
& \stackrel{\operatorname{sign}}{=}\left[\frac{\alpha_{i} p_{i}}{\bar{\alpha}_{p}}\left(\frac{\partial \bar{F}\left(t \mid \lambda_{i}\right)}{\partial \lambda_{i}}\right) \bar{F}^{\alpha_{i}-1}\left(t \mid \lambda_{i}\right)-\frac{\alpha_{j} p_{j}}{\bar{\alpha}_{p}}\left(\frac{\partial \bar{F}\left(t \mid \lambda_{j}\right)}{\partial \lambda_{j}}\right) \bar{F}^{\alpha_{j}-1}\left(t \mid \lambda_{j}\right)\right] \leq 0,
\end{aligned}
$$

as from condition $(i v), \alpha_{i} p_{i} \geq \alpha_{j} p_{j}$ and from condition $(i), \bar{F}\left(t \mid \lambda_{i}\right)$ is decreasing and convex, thus $-\frac{\partial \bar{F}\left(t \mid \lambda_{i}\right)}{\partial \lambda_{i}} \geq-\frac{\partial \bar{F}\left(t \mid \lambda_{j}\right)}{\partial \lambda_{j}} \geq 0$ and from condition $(i i), \bar{F}^{\alpha_{i}-1}\left(t \mid \lambda_{i}\right) \geq \bar{F}^{\alpha_{j}-1}\left(t \mid \lambda_{j}\right)$. Thus

$$
\left(\lambda_{i}-\lambda_{j}\right)\left(\frac{\partial \phi(\boldsymbol{\lambda})}{\partial \lambda_{i}}-\frac{\partial \phi(\boldsymbol{\lambda})}{\partial \lambda_{j}}\right) \geq 0
$$

Consequently, $\bar{F}_{W_{n}(\boldsymbol{p}, \boldsymbol{\lambda})}\left(t, \bar{\alpha}_{p}\right)$ is decreasing and Schur-convex for $\alpha_{i} \geq 1$. Now, condition (iii), $\boldsymbol{\lambda} \stackrel{w}{\succeq} \boldsymbol{\gamma}$, together with condition of Lemma 6.4 imply $\phi(\boldsymbol{\lambda}) \geq \phi(\gamma)$. This means $\bar{F}_{W_{n}(\boldsymbol{p}, \boldsymbol{\lambda})}\left(t, \bar{\alpha}_{p}\right) \geq$ $\bar{F}_{W_{n}(\boldsymbol{p}, \boldsymbol{\gamma})}\left(t, \bar{\alpha}_{p}\right)$ completing the proof of the theorem.

Remark 6.6. Theorem 6.5 extends the result of Theorem 3.2 in Nadeb and Torabi (2020) that compares two finite ordinary mixtures with respect to parameter $\lambda$.

To illustrate Theorem 6.5, we present a numerical example.

Example 6.7. Let $\bar{F}(t \mid \lambda)=e^{-\lambda t}$, for $t \in[0,+\infty)$. Clearly, $\bar{F}(t \mid \lambda)$ is decreasing and convex in $\lambda$. Set $\left(p_{1}, p_{2}, p_{3}\right)=(0.55,0.35,0.1),\left(\lambda_{1}, \lambda_{2}, \lambda_{3}\right)=(0.4,0.7,0.8),\left(\gamma_{1}, \gamma_{2}, \gamma_{3}\right)=(0.5,0.8,0.9)$ and $\left(\alpha_{1}, \alpha_{2}, \alpha_{3}\right)=(2.5,3,4)$. As we see, $\boldsymbol{\lambda} \succeq \boldsymbol{w} \gamma,(\boldsymbol{p}, \boldsymbol{\lambda}) \in \mathcal{U}_{3}$ and $(\boldsymbol{p}, \gamma) \in \mathcal{U}_{3}$ and $\left(\alpha_{1} p_{1}, \alpha_{2} p_{2}, \alpha_{3} p_{3}\right)=$ $(1.375,1.05,0.4)$. Thus all conditions of Theorem 6.5 are satisfied. 


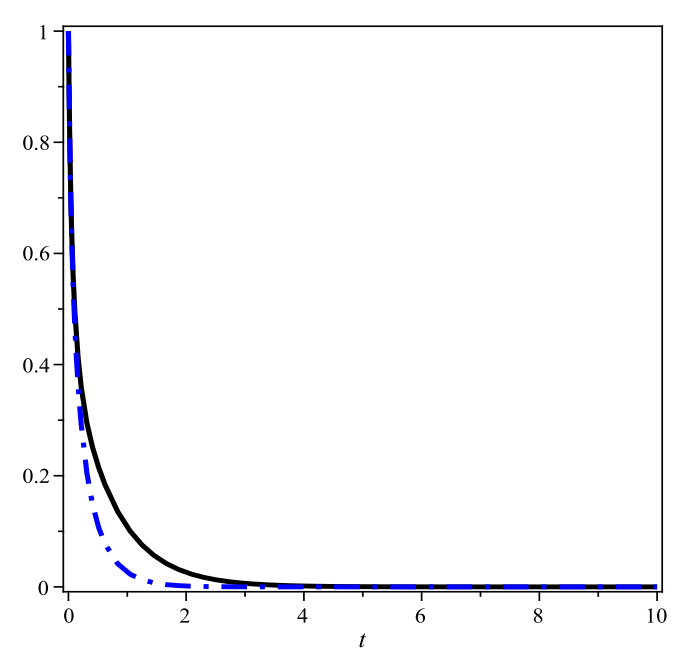

(a)

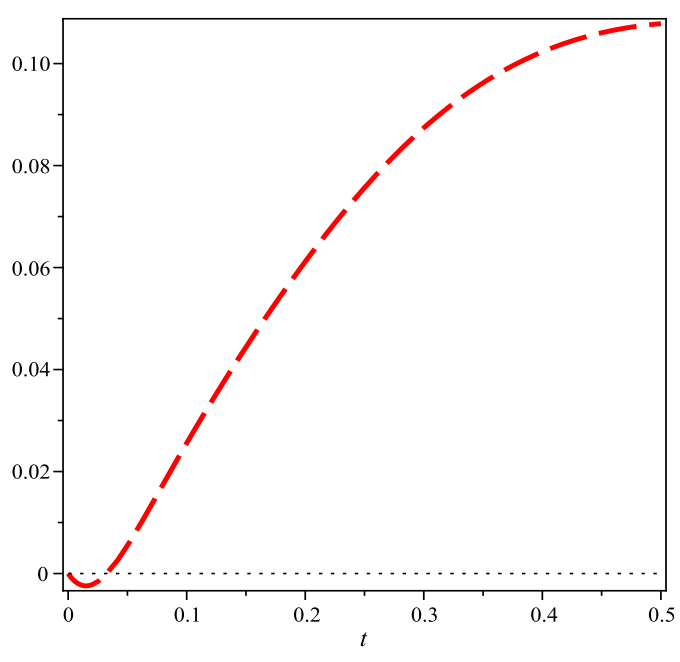

(b)

Figure 2: (a): The plots of $\left.\bar{F}_{W_{3}(\boldsymbol{p}, \boldsymbol{\lambda}}\right)\left(t, \bar{\alpha}_{p}\right)$ (solid) and $\left.\bar{F}_{W_{3}(\boldsymbol{p}, \boldsymbol{\gamma}}\right)\left(t, \bar{\alpha}_{p}\right)$ (dash dot). (b): $g_{1}(t)=$ $\left.\left.\bar{F}_{W_{3}(\boldsymbol{p}, \boldsymbol{\lambda}}\right)\left(t, \bar{\alpha}_{p}\right)-\bar{F}_{W_{3}(\boldsymbol{p}, \gamma}\right)\left(t, \bar{\alpha}_{p}\right)$.

However, if conditions $(\boldsymbol{p}, \boldsymbol{\lambda}) \in \mathcal{U}_{n}$ and $(\boldsymbol{p}, \boldsymbol{\gamma}) \in \mathcal{U}_{n}$ in Theorem 6.5 are removed, the result may be not true any more. The following counterexample illustrates this.

Counterexample 6.8. In Example 6.7, set $\left(\lambda_{1}, \lambda_{2}, \lambda_{3}\right)=(18,5,1)$ and $\left(\gamma_{1}, \gamma_{2}, \gamma_{3}\right)=(17,5,2)$. Thus $(\boldsymbol{p}, \boldsymbol{\lambda}) \notin \mathcal{U}_{3}$ and $(\boldsymbol{p}, \boldsymbol{\gamma}) \notin \mathcal{U}_{3}$. Figure 2 plots $\left.\left.\bar{F}_{W_{3}(\boldsymbol{p}, \boldsymbol{\lambda}}\right)\left(t, \bar{\alpha}_{p}\right), \bar{F}_{W_{3}(\boldsymbol{p}, \boldsymbol{\gamma}}\right)\left(t, \bar{\alpha}_{p}\right)$ and $g_{1}(t)=$ $\left.\left.\bar{F}_{W_{3}(\boldsymbol{p}, \boldsymbol{\lambda}}\right)\left(t, \bar{\alpha}_{p}\right)-\bar{F}_{W_{3}(\boldsymbol{p}, \boldsymbol{\gamma}}\right)\left(t, \bar{\alpha}_{p}\right)$, which indicates that the usual stochastic ordering does not hold between $\left.\bar{F}_{W_{3}(\boldsymbol{p}, \boldsymbol{\lambda}}\right)\left(t, \bar{\alpha}_{p}\right)$ and $\left.\bar{F}_{W_{3}(\boldsymbol{p}, \boldsymbol{\gamma}}\right)\left(t, \bar{\alpha}_{p}\right)$.

Theorem 6.9. Let $\left.\bar{F}_{W_{n}(\boldsymbol{p}, \boldsymbol{\lambda}}\right)\left(t, \bar{\alpha}_{p}\right)$ and $\bar{F}_{W_{n}(\boldsymbol{p}, \boldsymbol{\gamma})}\left(t, \bar{\alpha}_{p}\right)$, be two $n$-component generalized finite $\alpha$ mixtures with common mixing proportions $p_{1}, \ldots, p_{n}$ and parameters $\lambda_{1}, \ldots, \lambda_{n}$ and $\gamma_{1}, \ldots, \gamma_{n}$, respectively, where $\bar{\alpha}_{p}=\sum_{i=1}^{n} p_{i} \alpha_{i}$. Suppose that for $(\boldsymbol{p}, \boldsymbol{\lambda}) \in \mathcal{U}_{n}$ and $(\boldsymbol{p}, \boldsymbol{\gamma}) \in \mathcal{U}_{n}$ :

(i) $\bar{F}(t \mid \lambda)$ is increasing and concave in $\lambda>0$ for all $t \geq 0$;

(ii) $\alpha_{1} \leq \ldots, \leq \alpha_{n}$;

(iii) $\lambda \succeq \underbrace{w} \gamma$

Then for $\alpha_{i} \leq 0\left(0<\alpha_{i}<1\right.$ such that $\alpha_{i} p_{i} \geq \alpha_{j} p_{j}$ for all $\left.i \leq j, i, j=1,2, \ldots, n\right)$ :

$$
\bar{F}_{W_{n}(\boldsymbol{p}, \boldsymbol{\lambda})}\left(t, \bar{\alpha}_{p}\right) \leq \bar{F}_{W_{n}(\boldsymbol{p}, \boldsymbol{\gamma})}\left(t, \bar{\alpha}_{p}\right)
$$

The proof of this theorem is similar (with relevant alterations) to that of the previous one and therefore, is omitted. To illustrate the result, the following numerical example is provided.

Example 6.10. Let $\bar{F}(t \mid \lambda)=\left(\frac{\lambda}{\lambda+t^{2}}\right)^{a}$, for $t \in[0,+\infty)$, which is the survival function of the Compound Rayleigh distribution. Clearly, $\bar{F}(t \mid \lambda)$ is increasing and concave in $\lambda$ for $0<a<1$. 


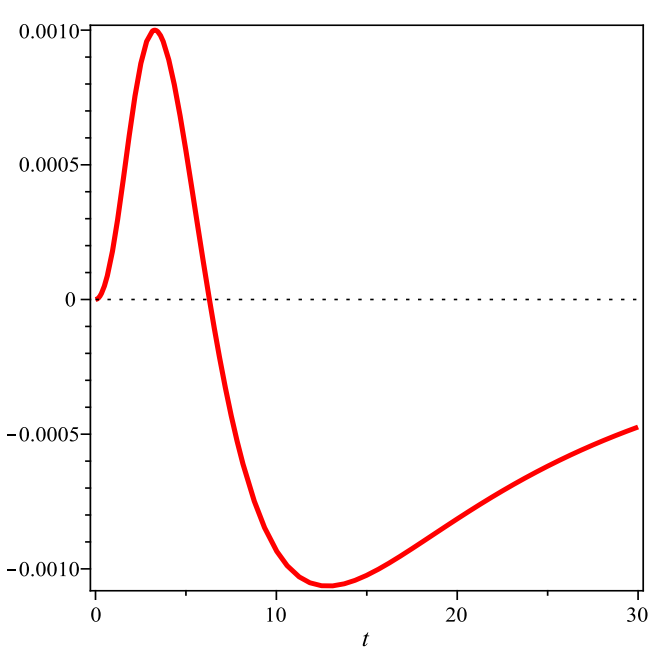

(a)

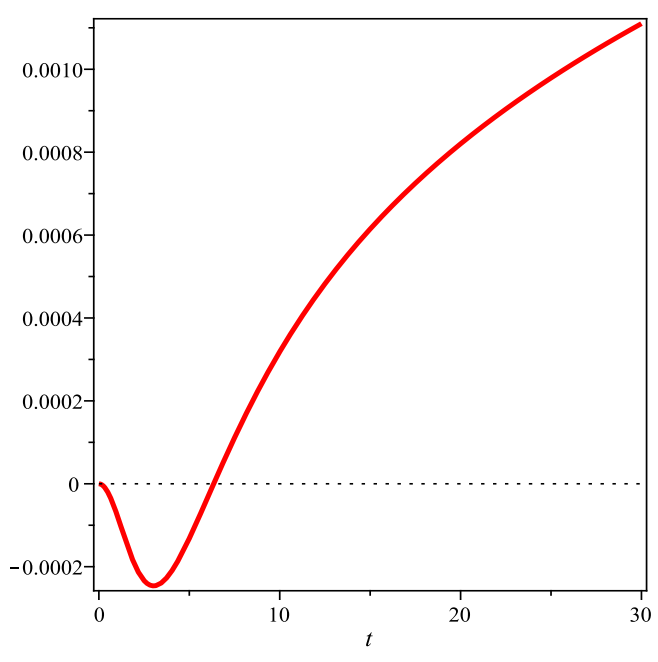

(b)

Figure 3: (a): $\left.\left.g_{2}(t)=\bar{F}_{W_{3}(\boldsymbol{p}, \boldsymbol{\gamma}}\right)\left(t, \bar{\alpha}_{p}\right)-\bar{F}_{W_{3}(\boldsymbol{p}, \boldsymbol{\lambda}}\right)\left(t, \bar{\alpha}_{p}\right)$ for $\alpha_{i} \leq 0$. (b): $\left.g_{2}(t)=\bar{F}_{W_{3}(\boldsymbol{p}, \boldsymbol{\gamma}}\right)\left(t, \bar{\alpha}_{p}\right)-$ $\left.\bar{F}_{W_{3}(\boldsymbol{p}, \boldsymbol{\lambda}}\right)\left(t, \bar{\alpha}_{p}\right)$ for $0<\alpha_{i}<1$.

For $\alpha_{i} \leq 0\left(0<\alpha_{i}<1\right)$, set $a=0.9,\left(p_{1}, p_{2}, p_{3}\right)=(0.6,0.3,0.1),\left(\lambda_{1}, \lambda_{2}, \lambda_{3}\right)=(10,12,14)$, $\left(\gamma_{1}, \gamma_{2}, \gamma_{3}\right)=(11,12,15)$ and $\left(\alpha_{1}, \alpha_{2}, \alpha_{3}\right)=(-4,-3,-2.5)\left(\left(\alpha_{1}, \alpha_{2}, \alpha_{3}\right)=(0.4,0.5,0.6)\right)$. As we see, $\boldsymbol{\lambda} \succeq \boldsymbol{w} \gamma,(\boldsymbol{p}, \boldsymbol{\lambda}) \in \mathcal{U}_{3}$ and $(\boldsymbol{p}, \gamma) \in \mathcal{U}_{3}$ and for $0<\alpha_{i}<1,\left(\alpha_{1} p_{1}, \alpha_{2} p_{2}, \alpha_{3} p_{3}\right)=(0.24,0.15,0.06)$. Thus, all conditions of Theorem 6.9 are satisfied.

In the following counterexample, we show that conditions $(\boldsymbol{p}, \boldsymbol{\lambda}) \in \mathcal{U}_{n}$ and $(\boldsymbol{p}, \boldsymbol{\gamma}) \in \mathcal{U}_{n}$ in Theorem 6.9 can not be dropped.

Counterexample 6.11. In Example 6.10, set $\left(\lambda_{1}, \lambda_{2}, \lambda_{3}\right)=(20,10.5,10)$ and $\left(\gamma_{1}, \gamma_{2}, \gamma_{3}\right)=(19.5,11,10)$. Thus $(\boldsymbol{p}, \boldsymbol{\lambda}) \notin \mathcal{U}_{3}$ and $(\boldsymbol{p}, \gamma) \notin \mathcal{U}_{3}$. Figure 3 plots $\left.\left.g_{2}(t)=\bar{F}_{W_{3}(\boldsymbol{p}, \boldsymbol{\gamma}}\right)\left(t, \bar{\alpha}_{p}\right)-\bar{F}_{W_{3}(\boldsymbol{p}, \boldsymbol{\lambda}}\right)\left(t, \bar{\alpha}_{p}\right)$ for $\alpha_{i} \leq 0$ and $0<\alpha_{i}<1$, which indicates that the usual stochastic ordering does not hold between $\left.\bar{F}_{W_{3}(\boldsymbol{p}, \boldsymbol{\lambda}}\right)\left(t, \bar{\alpha}_{p}\right)$ and $\left.\bar{F}_{W_{3}(\boldsymbol{p}, \boldsymbol{\gamma}}\right)\left(t, \bar{\alpha}_{p}\right)$.

Corollary 6.12. Let $\bar{F}(t \mid \lambda)$ belongs to the proportional hazard family, $\bar{F}(t \mid \lambda)=\bar{F}^{\lambda}(t)$, for all $t$, where $\bar{F}(t)$ is the baseline survival function. Then, for $\alpha_{i} \geq 1, i=1,2, \ldots, n$ such that $\alpha_{1} \leq \alpha_{2} \leq$ $\cdots \leq \alpha_{n}$ and $\alpha_{i} p_{i} \geq \alpha_{j} p_{j}$ for all $i \leq j, i, j=1,2, \ldots, n, \boldsymbol{\lambda} \succeq \boldsymbol{w} \gamma,(\boldsymbol{p}, \boldsymbol{\lambda}) \in \mathcal{U}_{n}$ and $(\boldsymbol{p}, \gamma) \in \mathcal{U}_{n}$, we have:

$$
\left.\left.\bar{F}_{W_{n}(\boldsymbol{p}, \boldsymbol{\lambda}}\right)\left(t, \bar{\alpha}_{p}\right) \geq \bar{F}_{W_{n}(\boldsymbol{p}, \boldsymbol{\gamma}}\right)\left(t, \bar{\alpha}_{p}\right) .
$$

Corollary 6.13. Let $\bar{F}(t \mid \lambda)$ belong to the accelerated lifetime (scale model) family, $\bar{F}(t \mid \lambda)=\bar{F}(\lambda t)$, for all $t$, where $\bar{F}(t)$ is the baseline survival function. Also, let $f(t)$ denoting the baseline density function, is decreasing in $t$. Then, for $\alpha_{i} \geq 1, i=1,2, \ldots, n$ such that $\alpha_{1} \leq \alpha_{2} \leq \cdots \leq \alpha_{n}$ and $\alpha_{i} p_{i} \geq \alpha_{j} p_{j}$ for all $i \leq j, i, j=1,2, \ldots, n, \boldsymbol{\lambda} \succeq \boldsymbol{w},(\boldsymbol{p}, \boldsymbol{\lambda}) \in \mathcal{U}_{n}$ and $(\boldsymbol{p}, \boldsymbol{\gamma}) \in \mathcal{U}_{n}$, we have:

$$
\left.\left.\bar{F}_{W_{n}(\boldsymbol{p}, \boldsymbol{\lambda}}\right)\left(t, \bar{\alpha}_{p}\right) \geq \bar{F}_{W_{n}(\boldsymbol{p}, \boldsymbol{\gamma}}\right)\left(t, \bar{\alpha}_{p}\right) .
$$


Corollary 6.14. Let $\bar{F}(t \mid \lambda)$ belong to the proportional reversed hazard family, $\bar{F}(t \mid \lambda)=1-F^{\lambda}(t)$, for all $t$, where $F(t)$ is the baseline distribution function. Then, for $\alpha_{i} \leq 0\left(0<\alpha_{i}<1\right.$ such that $\alpha_{i} p_{i} \geq \alpha_{j} p_{j}$ for all $\left.i \leq j, i, j=1,2, \ldots, n\right), i=1,2, \ldots, n$ such that $\alpha_{1} \leq \alpha_{2} \leq \cdots \leq \alpha_{n}, \lambda \succeq \gamma$, $(\boldsymbol{p}, \boldsymbol{\lambda}) \in \mathcal{U}_{n}$ and $(\boldsymbol{p}, \boldsymbol{\gamma}) \in \mathcal{U}_{n}$, we have:

$$
\left.\left.\bar{F}_{W_{n}(\boldsymbol{p}, \boldsymbol{\lambda}}\right)\left(t, \bar{\alpha}_{p}\right) \leq \bar{F}_{W_{n}(\boldsymbol{p}, \gamma}\right)\left(t, \bar{\alpha}_{p}\right) .
$$

From Theorems 6.5 and 6.9, we can obtain a lower or upper bound for the generalized finite $\alpha$-mixture as discussed in the following remark.

Remark 6.15. Set $\left(\gamma_{1}, \ldots, \gamma_{n}\right)=(\bar{\lambda}, \ldots, \bar{\lambda})$, where $\bar{\lambda}=\frac{1}{n} \sum_{i=1}^{n} \lambda_{i}$. It is easy to see that $\boldsymbol{\lambda} \stackrel{w}{\complement}^{w}$. Then, for $(\boldsymbol{p}, \boldsymbol{\lambda}) \in \mathcal{U}_{n}$ :

(i) If $\bar{F}(t \mid \lambda)$ is decreasing and convex in $\lambda>0$ for all $t$ and for $\alpha_{i} \geq 1, i=1,2, \ldots, n$ such that $\alpha_{1} \leq \alpha_{2} \leq \cdots \leq \alpha_{n}$ and $\alpha_{i} p_{i} \geq \alpha_{j} p_{j}$ for all $i \leq j, i, j=1,2, \ldots, n$, we have the following lower bound for the generalized finite $\alpha$-mixture:

$$
\left.\bar{F}_{W_{n}(\boldsymbol{p}, \boldsymbol{\lambda}}\right)\left(t, \bar{\alpha}_{p}\right) \geq \bar{F}^{\frac{\alpha_{n}}{\bar{\alpha}_{p}}}(t \mid \bar{\lambda})
$$

(ii) If $\bar{F}(t \mid \lambda)$ is increasing and concave in $\lambda>0$ for all $t$ and for $\alpha_{i} \leq 0\left(0<\alpha_{i}<1\right.$ such that $\alpha_{i} p_{i} \geq \alpha_{j} p_{j}$ for all $\left.i \leq j, i, j=1,2, \ldots, n\right), i=1,2, \ldots, n$ such that $\alpha_{1} \leq \alpha_{2} \leq \cdots \leq \alpha_{n}$, we have the following upper bound for the generalized finite $\alpha$-mixture:

$$
\left.\bar{F}_{W_{n}(\boldsymbol{p}, \boldsymbol{\lambda}}\right)\left(t, \bar{\alpha}_{p}\right) \leq \bar{F}^{\frac{\alpha_{n}}{\bar{\alpha}_{p}}}(t \mid \bar{\lambda})
$$

The next numerical examples illustrate Remark 6.15 for two cases $\alpha_{i} \geq 1$ and $\alpha_{i} \leq 0$.

Example 6.16. (i) Consider the Example 6.7. Let $\bar{F}(t \mid \lambda)=e^{-\lambda t}$, for $t \in[0,+\infty)$. Clearly, $\bar{F}(t \mid \lambda)$ is decreasing and convex in $\lambda$. Set $\left(p_{1}, p_{2}, p_{3}\right)=(0.55,0.35,0.1),\left(\lambda_{1}, \lambda_{2}, \lambda_{3}\right)=$ $(0.4,0.7,0.8),\left(\gamma_{1}, \gamma_{2}, \gamma_{3}\right)=(\bar{\lambda}, \bar{\lambda}, \bar{\lambda})=(0.63,0.63,0.63)$ and $\left(\alpha_{1}, \alpha_{2}, \alpha_{3}\right)=(2.5,3,4)$. As we see, $\boldsymbol{\lambda} \succeq \boldsymbol{w} \gamma,(\boldsymbol{p}, \boldsymbol{\lambda}) \in \mathcal{U}_{3}$ and $(\boldsymbol{p}, \overline{\boldsymbol{\lambda}}) \in \mathcal{U}_{3}$ and $\left(\alpha_{1} p_{1}, \alpha_{2} p_{2}, \alpha_{3} p_{3}\right)=(1.375,1.05,0.4)$. Thus, all conditions of Remark $6.15(i)$ are satisfied.

(ii) Consider the Example 6.10. Let $\bar{F}(t \mid \lambda)=\left(\frac{\lambda}{\lambda+t^{2}}\right)^{a}$, for $t \in[0,+\infty)$, which is the survival function of the Compound Rayleigh distribution. Clearly, $\bar{F}(t \mid \lambda)$ is increasing and concave in $\lambda$ for $0<a<1$. For $\alpha_{i} \leq 0$, set $a=0.9,\left(p_{1}, p_{2}, p_{3}\right)=(0.6,0.3,0.1),\left(\lambda_{1}, \lambda_{2}, \lambda_{3}\right)=(10,12,14)$, $\left(\gamma_{1}, \gamma_{2}, \gamma_{3}\right)=(\bar{\lambda}, \bar{\lambda}, \bar{\lambda})=(12,12,12)$ and $\left(\alpha_{1}, \alpha_{2}, \alpha_{3}\right)=(-4,-3,-2.5)$. As we see, $\boldsymbol{\lambda} \succeq \boldsymbol{w}$, $(\boldsymbol{p}, \boldsymbol{\lambda}) \in \mathcal{U}_{3}$ and $(\boldsymbol{p}, \overline{\boldsymbol{\lambda}}) \in \mathcal{U}_{3}$. Thus, all conditions of Remark 6.15 (ii) are satisfied.

\subsection{Hazard rate order}

In this subsection, we consider the 2-component generalized finite $\alpha$-mixture and provide some comparisons in the sense of the hazard rate order. 
Theorem 6.17. Let

$$
\left.\bar{F}_{W_{2}(\boldsymbol{p}, \boldsymbol{\lambda}}\right)\left(t, \bar{\alpha}_{p}\right)=\left[\sum_{i=1}^{2} p_{i} \bar{F}^{\alpha_{i}}\left(t \mid \lambda_{i}\right)\right]^{1 / \bar{\alpha}_{p}}
$$

and

$$
\bar{F}_{W_{2}(\boldsymbol{p}, \gamma)}\left(t, \bar{\alpha}_{p}\right)=\left[\sum_{i=1}^{2} p_{i} \bar{F}^{\alpha_{i}}\left(t \mid \gamma_{i}\right)\right]^{1 / \bar{\alpha}_{p}},
$$

be two 2-component generalized finite $\alpha$-mixtures with common mixing proportions $p_{1}, p_{2}$ and parameters $\lambda_{1}, \lambda_{2}$ and $\gamma_{1}, \gamma_{2}$, respectively, where $\bar{\alpha}_{p}=\sum_{i=1}^{2} p_{i} \alpha_{i}$. Suppose that for $(\boldsymbol{p}, \boldsymbol{\lambda}) \in \mathcal{U}_{2}$ and $(\boldsymbol{p}, \gamma) \in \mathcal{U}_{2}$ :

(i) $r(t \mid \lambda)$ is increasing (decreasing) and concave (convex) in $\lambda>0$ for all $t$;

(ii) $\bar{F}(t \mid \lambda)$ is decreasing (increasing) in $\lambda>0$ for all $t$;

(iii) $\alpha_{1} \leq \alpha_{2}$;

(iv) $\lambda \stackrel{m}{\succeq} \gamma$

Then for $\alpha_{i} \geq 0$ such that $\alpha_{1} p_{1} \geq \alpha_{2} p_{2}\left(\alpha_{i}<0\right)$ :

$$
r_{W_{2}(\boldsymbol{p}, \boldsymbol{\lambda})}\left(t, \bar{\alpha}_{p}\right) \leq(\geq) r_{W_{2}(\boldsymbol{p}, \boldsymbol{\gamma})}\left(t, \bar{\alpha}_{p}\right),
$$

where $r_{W_{2}(\boldsymbol{p}, \boldsymbol{\lambda})}\left(t, \bar{\alpha}_{p}\right)$ and $r_{W_{2}(\boldsymbol{p}, \boldsymbol{\gamma})}\left(t, \bar{\alpha}_{p}\right)$ are the hazard rate functions that correspond to $\left.\bar{F}_{W_{2}(\boldsymbol{p}, \boldsymbol{\lambda}}\right)\left(t, \bar{\alpha}_{p}\right)$ and $\left.\bar{F}_{W_{2}(\boldsymbol{p}, \gamma}\right)\left(t, \bar{\alpha}_{p}\right)$, respectively.

Proof. Without loss of generality, suppose that $p_{1} \geq p_{2}$. From assumption $(\boldsymbol{p}, \boldsymbol{\lambda}) \in \mathcal{U}_{2}$ and $(\boldsymbol{p}, \gamma) \in \mathcal{U}_{2}$, we have $0<\lambda_{1} \leq \lambda_{2}$ and $0<\gamma_{1} \leq \gamma_{2}$. The hazard rate function of $\left.\bar{F}_{W_{2}(\boldsymbol{p}, \boldsymbol{\lambda}}\right)\left(t, \bar{\alpha}_{p}\right)$ is:

$$
\begin{aligned}
r_{W_{2}(\boldsymbol{p}, \boldsymbol{\lambda})}\left(t, \bar{\alpha}_{p}\right) & =\frac{1}{\bar{\alpha}_{p}}\left[\frac{\sum_{i=1}^{2} p_{i} \alpha_{i} r\left(t \mid \lambda_{i}\right) \bar{F}^{\alpha_{i}}\left(t \mid \lambda_{i}\right)}{\sum_{i=1}^{2} p_{i} \bar{F}^{\alpha_{i}}\left(t \mid \lambda_{i}\right)}\right] \\
& =\frac{1}{\bar{\alpha}_{p}}\left[\frac{p_{1} \alpha_{1} r\left(t \mid \lambda_{1}\right) \bar{F}^{\alpha_{1}}\left(t \mid \lambda_{1}\right)+p_{2} \alpha_{2} r\left(t \mid \lambda_{2}\right) \bar{F}^{\alpha_{2}}\left(t \mid \lambda_{2}\right)}{p_{1} \bar{F}^{\alpha_{1}}\left(t \mid \lambda_{1}\right)+p_{2} \bar{F}^{\alpha_{2}}\left(t \mid \lambda_{2}\right)}\right] \\
& =\frac{1}{\bar{\alpha}_{p}}\left[\frac{\Psi_{1}(t ; \boldsymbol{p}, \boldsymbol{\lambda})}{\Psi_{2}(t ; \boldsymbol{p}, \boldsymbol{\lambda})}\right] .
\end{aligned}
$$


Thus,

$$
\begin{aligned}
\frac{\partial r_{W_{2}(\boldsymbol{p}, \boldsymbol{\lambda})}\left(t, \bar{\alpha}_{p}\right)}{\partial \lambda_{1}} & =\frac{1}{\bar{\alpha}_{p}}\left[\frac{\frac{\partial \Psi_{1}(t ; \boldsymbol{p}, \boldsymbol{\lambda})}{\partial \lambda_{1}} \Psi_{2}(t ; \boldsymbol{p}, \boldsymbol{\lambda})-\frac{\partial \Psi_{2}(t ; \boldsymbol{p}, \boldsymbol{\lambda})}{\partial \lambda_{1}} \Psi_{1}(t ; \boldsymbol{p}, \boldsymbol{\lambda})}{\Psi_{2}^{2}(t ; \boldsymbol{p}, \boldsymbol{\lambda})}\right] \\
& \stackrel{\operatorname{sig} n}{=} \frac{1}{\bar{\alpha}_{p}}\left[p_{1} \alpha_{1}\left(\frac{\partial r\left(t \mid \lambda_{1}\right)}{\partial \lambda_{1}} \bar{F}^{\alpha_{1}}\left(t \mid \lambda_{1}\right)+\alpha_{1} \frac{\partial \bar{F}\left(t \mid \lambda_{1}\right)}{\partial \lambda_{1}} \bar{F}^{\alpha_{1}-1}\left(t \mid \lambda_{1}\right) r\left(t \mid \lambda_{1}\right)\right) \Psi_{2}(t ; \boldsymbol{p}, \boldsymbol{\lambda})\right. \\
& \left.-\left(p_{1} \alpha_{1} \frac{\partial \bar{F}\left(t \mid \lambda_{1}\right)}{\partial \lambda_{1}} \bar{F}^{\alpha_{1}-1}\left(t \mid \lambda_{1}\right)\right) \Psi_{1}(t ; \boldsymbol{p}, \boldsymbol{\lambda})\right] \\
& =\frac{p_{1} \alpha_{1}}{\bar{\alpha}_{p}}\left[\frac{\partial r\left(t \mid \lambda_{1}\right)}{\partial \lambda_{1}} \bar{F}^{\alpha_{1}}\left(t \mid \lambda_{1}\right) \Psi_{2}(t ; \boldsymbol{p}, \boldsymbol{\lambda})+\alpha_{1} \frac{\partial \bar{F}\left(t \mid \lambda_{1}\right)}{\partial \lambda_{1}} \bar{F}^{\alpha_{1}-1}\left(t \mid \lambda_{1}\right) r\left(t \mid \lambda_{1}\right) \Psi_{2}(t ; \boldsymbol{p}, \boldsymbol{\lambda})\right. \\
& \left.-\frac{\partial \bar{F}\left(t \mid \lambda_{1}\right)}{\partial \lambda_{1}} \bar{F}^{\alpha_{1}-1}\left(t \mid \lambda_{1}\right) \Psi_{1}(t ; \boldsymbol{p}, \boldsymbol{\lambda})\right] \\
& =\frac{p_{1} \alpha_{1}\left[\frac{\partial r\left(t \mid \lambda_{1}\right)}{\partial \lambda_{1}} \bar{F}^{\alpha_{1}}\left(t \mid \lambda_{1}\right) \Psi_{2}(t ; \boldsymbol{p}, \boldsymbol{\lambda})\right.}{\bar{\alpha}_{p}}\left[\frac{\partial \bar{F}\left(t \mid \lambda_{1}\right)}{\partial \lambda_{1}} \bar{F}^{\alpha_{1}-1}\left(t \mid \lambda_{1}\right)\left(\alpha_{1} r\left(t \mid \lambda_{1}\right) \Psi_{2}(t ; \boldsymbol{p}, \boldsymbol{\lambda})-\Psi_{1}(t ; \boldsymbol{p}, \boldsymbol{\lambda})\right)\right] \\
& =\frac{p_{1} \alpha_{1}\left[\frac{\partial r\left(t \mid \lambda_{1}\right)}{\partial \lambda_{1}} \bar{F}^{\alpha_{1}}\left(t \mid \lambda_{1}\right) \Psi_{2}(t ; \boldsymbol{p}, \boldsymbol{\lambda})\right.}{\bar{\alpha}_{p}} \\
& \left.+\frac{\partial \bar{F}\left(t \mid \lambda_{1}\right)}{\partial \lambda_{1}} \bar{F}^{\alpha_{1}-1}\left(t \mid \lambda_{1}\right)\left(p_{2} \bar{F}^{\alpha_{2}}\left(t \mid \lambda_{2}\right)\left(\alpha_{1} r\left(t \mid \lambda_{1}\right)-\alpha_{2} r\left(t \mid \lambda_{2}\right)\right)\right)\right] .
\end{aligned}
$$

Now, we have

$$
\begin{aligned}
& \frac{\partial r_{W_{2}(\boldsymbol{p}, \boldsymbol{\lambda})}\left(t, \bar{\alpha}_{p}\right)}{\partial \lambda_{1}}-\frac{\partial r_{W_{2}(\boldsymbol{p}, \boldsymbol{\lambda})}\left(t, \bar{\alpha}_{p}\right)}{\partial \lambda_{2}} \\
& \stackrel{\operatorname{sign}}{=} \frac{1}{\bar{\alpha}_{p}}\left[\left(p_{1} \alpha_{1} \frac{\partial r\left(t \mid \lambda_{1}\right)}{\partial \lambda_{1}} \bar{F}^{\alpha_{1}}\left(t \mid \lambda_{1}\right)-p_{2} \alpha_{2} \frac{\partial r\left(t \mid \lambda_{2}\right)}{\partial \lambda_{2}} \bar{F}^{\alpha_{2}}\left(t \mid \lambda_{2}\right)\right) \Psi_{2}(t ; \boldsymbol{p}, \boldsymbol{\lambda})\right] \\
& +\frac{1}{\bar{\alpha}_{p}}\left[p_{1} \alpha_{1} \frac{\partial \bar{F}\left(t \mid \lambda_{1}\right)}{\partial \lambda_{1}} \bar{F}^{\alpha_{1}-1}\left(t \mid \lambda_{1}\right)\left(p_{2} \bar{F}^{\alpha_{2}}\left(t \mid \lambda_{2}\right)\left(\alpha_{1} r\left(t \mid \lambda_{1}\right)-\alpha_{2} r\left(t \mid \lambda_{2}\right)\right)\right)\right. \\
& \left.-p_{2} \alpha_{2} \frac{\partial \bar{F}\left(t \mid \lambda_{2}\right)}{\partial \lambda_{2}} \bar{F}^{\alpha_{2}-1}\left(t \mid \lambda_{2}\right)\left(p_{1} \bar{F}^{\alpha_{1}}\left(t \mid \lambda_{1}\right)\left(\alpha_{2} r\left(t \mid \lambda_{2}\right)-\alpha_{1} r\left(t \mid \lambda_{1}\right)\right)\right)\right] \\
& =\frac{1}{\bar{\alpha}_{p}}[A+B-C]
\end{aligned}
$$

where,

$$
\begin{gathered}
A=\left(p_{1} \alpha_{1} \frac{\partial r\left(t \mid \lambda_{1}\right)}{\partial \lambda_{1}} \bar{F}^{\alpha_{1}}\left(t \mid \lambda_{1}\right)-p_{2} \alpha_{2} \frac{\partial r\left(t \mid \lambda_{2}\right)}{\partial \lambda_{2}} \bar{F}^{\alpha_{2}}\left(t \mid \lambda_{2}\right)\right) \Psi_{2}(t ; \boldsymbol{p}, \boldsymbol{\lambda}), \\
B=p_{1} \alpha_{1} \frac{\partial \bar{F}\left(t \mid \lambda_{1}\right)}{\partial \lambda_{1}} \bar{F}^{\alpha_{1}-1}\left(t \mid \lambda_{1}\right)\left(p_{2} \bar{F}^{\alpha_{2}}\left(t \mid \lambda_{2}\right)\left(\alpha_{1} r\left(t \mid \lambda_{1}\right)-\alpha_{2} r\left(t \mid \lambda_{2}\right)\right)\right)
\end{gathered}
$$

and

$$
C=p_{2} \alpha_{2} \frac{\partial \bar{F}\left(t \mid \lambda_{2}\right)}{\partial \lambda_{2}} \bar{F}^{\alpha_{2}-1}\left(t \mid \lambda_{2}\right)\left(p_{1} \bar{F}^{\alpha_{1}}\left(t \mid \lambda_{1}\right)\left(\alpha_{2} r\left(t \mid \lambda_{2}\right)-\alpha_{1} r\left(t \mid \lambda_{1}\right)\right)\right) .
$$


From condition (ii), $\bar{F}\left(t \mid \lambda_{1}\right) \geq(\leq) \bar{F}\left(t \mid \lambda_{2}\right)$. Since, from condition (iii), $\alpha_{1} \leq \alpha_{2}, \bar{F}^{\alpha_{1}}\left(t \mid \lambda_{1}\right) \geq$ $\bar{F}^{\alpha_{2}}\left(t \mid \lambda_{2}\right)$. From condition $(i), \frac{\partial r\left(t \mid \lambda_{1}\right)}{\partial \lambda_{1}} \geq(\leq) \frac{\partial r\left(t \mid \lambda_{2}\right)}{\partial \lambda_{2}} \geq(\leq) 0$. Consequently, $A \geq 0$ follows from condition $\alpha_{1} p_{1} \geq \alpha_{2} p_{2}$. Also, we claim that $B \geq 0$, because from condition $(i), r\left(t \mid \lambda_{1}\right) \leq(\geq) r\left(t \mid \lambda_{2}\right)$, and condition (iii), $\alpha_{1} \leq \alpha_{2},\left(\alpha_{1} r\left(t \mid \lambda_{1}\right)-\alpha_{2} r\left(t \mid \lambda_{2}\right)\right) \leq 0$. On the other hand, from condition (ii), $\frac{\partial \bar{F}\left(t \mid \lambda_{1}\right)}{\partial \lambda_{1}} \leq(\geq) 0$. Consequently, $B \geq 0$. Similarly, $C \leq 0$ and hence $-C \geq 0$. Therefore,

$$
\left(\lambda_{1}-\lambda_{2}\right)\left(\frac{\partial r_{W_{2}(\boldsymbol{p}, \boldsymbol{\lambda})}\left(t, \overline{\alpha_{p}}\right)}{\partial \lambda_{1}}-\frac{\partial r_{W_{2}(\boldsymbol{p}, \boldsymbol{\lambda})}\left(t, \overline{\alpha_{p}}\right)}{\partial \lambda_{2}}\right) \leq(\geq) 0
$$

Consequently, according to Lemma $6.3, r_{W_{2}(\boldsymbol{p}, \boldsymbol{\lambda})}\left(t, \overline{\alpha_{p}}\right)$ is Schur-concave (Schur-convex). Using condition $(i v), \boldsymbol{\lambda} \stackrel{m}{\succeq} \boldsymbol{\gamma}$, we have: $r_{W_{2}(\boldsymbol{p}, \boldsymbol{\lambda})}\left(t, \bar{\alpha}_{p}\right) \leq(\geq) r_{W_{2}(\boldsymbol{p}, \boldsymbol{\gamma})}\left(t, \bar{\alpha}_{p}\right)$.

This completes the proof.

Remark 6.18. Theorem 6.17 is proved for $n=2$. We have not yet been able to prove whether the result holds for $n>2$ or not leaving it as an open problem for now (see also Section 4 of Hazra and Finkelstein (2018) and Nadeb and Torabi (2020), where the authors make the similar statement for ordinary mixtures when $n>2$ ).

Theorem 6.17 is illustrated by the following numerical example:

Example 6.19. Let $\bar{F}(t \mid \lambda)=e^{-\lambda t}$, for $t \in[0,+\infty)$. Clearly, $\bar{F}(t \mid \lambda)$ is decreasing and $r(t \mid \lambda)=$ $\lambda$ is increasing and concave in $\lambda$. For $\alpha_{i} \geq 0$, set $\left(p_{1}, p_{2}\right)=(0.55,0.45),\left(\lambda_{1}, \lambda_{2}\right)=(0.4,0.7)$, $\left(\gamma_{1}, \gamma_{2}\right)=(0.5,0.6)$ and $\left(\alpha_{1}, \alpha_{2}\right)=(2.5,3)$. As we see, $\boldsymbol{\lambda} \succeq \boldsymbol{m} \boldsymbol{\gamma},(\boldsymbol{p}, \boldsymbol{\lambda}) \in \mathcal{U}_{2}$ and $(\boldsymbol{p}, \boldsymbol{\gamma}) \in \mathcal{U}_{2}$ and $\left(\alpha_{1} p_{1}, \alpha_{2} p_{2}\right)=(1.375,1.35)$. Thus, all conditions of Theorem 6.17 are satisfied.

In the following counterexample, we show that conditions $(\boldsymbol{p}, \boldsymbol{\lambda}) \in \mathcal{U}_{2}$ and $(\boldsymbol{p}, \gamma) \in \mathcal{U}_{2}$ in Theorem 6.17 can not be dropped.

Counterexample 6.20. In Example 6.19, set $\left(\lambda_{1}, \lambda_{2}\right)=(0.8,0.5),\left(\gamma_{1}, \gamma_{2}\right)=(0.7,0.6)$. As we see, $\boldsymbol{\lambda} \stackrel{m}{\succeq} \boldsymbol{\gamma}$, but $(\boldsymbol{p}, \boldsymbol{\lambda}) \notin \mathcal{U}_{2}$ and $(\boldsymbol{p}, \gamma) \notin \mathcal{U}_{2}$. Figure 4 plots $r_{W_{2}(\boldsymbol{p}, \boldsymbol{\lambda})}\left(t, \bar{\alpha}_{p}\right), r_{W_{2}(\boldsymbol{p}, \boldsymbol{\gamma})}\left(t, \bar{\alpha}_{p}\right)$ and $g_{3}(t)=r_{W_{2}(\boldsymbol{p}, \boldsymbol{\gamma})}\left(t, \bar{\alpha}_{p}\right)-r_{W_{2}(\boldsymbol{p}, \boldsymbol{\lambda})}\left(t, \bar{\alpha}_{p}\right)$ for $\alpha_{i} \geq 0$, which indicates that the hazard rate ordering does not hold between $\left.\bar{F}_{W_{2}(\boldsymbol{p}, \boldsymbol{\lambda}}\right)\left(t, \bar{\alpha}_{p}\right)$ and $\left.\bar{F}_{W_{2}(\boldsymbol{p}, \boldsymbol{\gamma}}\right)\left(t, \bar{\alpha}_{p}\right)$.

The result of the next corollary extends the result of Theorem 4.2 in Nadeb and Torabi (2020), which compares two finite ordinary mixtures with respect to parameter $\lambda$ in the generalized finite $\alpha$-mixture. Its proof directly follows from Theorem 6.17 and therefore, is omitted.

Corollary 6.21. Let $\bar{F}(t \mid \lambda)$ belong to the proportional hazards family, $\bar{F}(t \mid \lambda)=\bar{F}^{\lambda}(t)$, for all $t$, where $\bar{F}(t)$ is the baseline survival function. Then, for $0 \leq \alpha_{1} \leq \alpha_{2}$ such that $\alpha_{1} p_{1} \geq \alpha_{2} p_{2}, \lambda \succeq \underbrace{m}$, $(\boldsymbol{p}, \boldsymbol{\lambda}) \in \mathcal{U}_{2}$ and $(\boldsymbol{p}, \gamma) \in \mathcal{U}_{2}$, we have:

$$
\left.\left.r_{W_{2}(\boldsymbol{p}, \boldsymbol{\lambda}}\right)\left(t, \bar{\alpha}_{p}\right) \leq r_{W_{2}(\boldsymbol{p}, \boldsymbol{\gamma}}\right)\left(t, \bar{\alpha}_{p}\right) .
$$

Remark 6.22. The results in this paper are obtained under the assumption that $\alpha_{i} \neq 0$. It should be pointed out that the case $\alpha_{i}=0$ can be considered as a special case of ordinary finite $\alpha$-mixtures (see, the model (2)). For this special case, we refer to Asadi et al. (2019) for Theorems 3.1 and 3.5 and Lemma 3.3; for results given in sections 3, 4, 5 etc, we refer to Shojaee et al. (2021) and for results given in section 6, we refer to Barmalzan et al. (2021). 


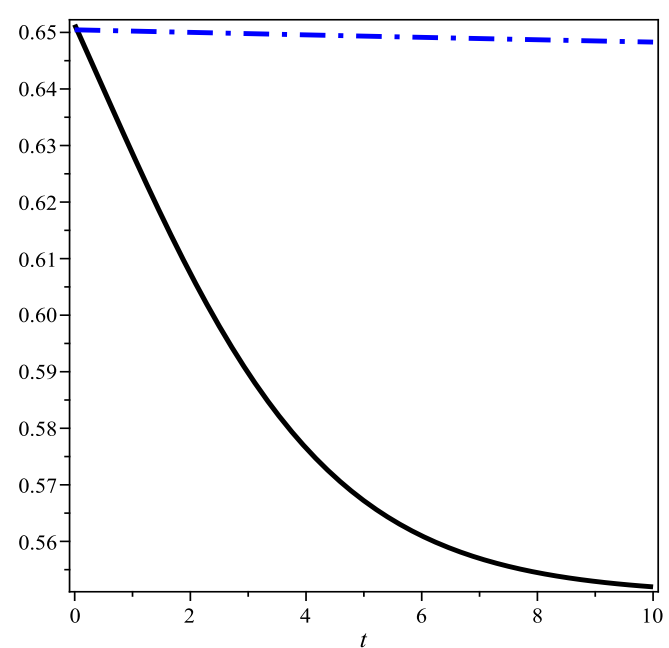

(a)

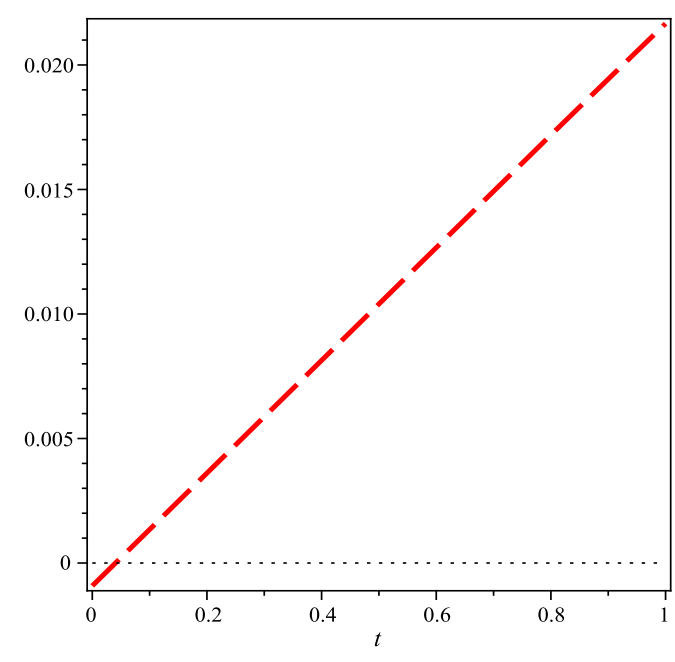

(b)

Figure 4: (a): $r_{W_{2}(\boldsymbol{p}, \boldsymbol{\lambda})}\left(t, \bar{\alpha}_{p}\right)$ (solid) and $r_{W_{2}(\boldsymbol{p}, \boldsymbol{\gamma})}\left(t, \bar{\alpha}_{p}\right)$ (dash dot). (b): $g_{3}(t)=r_{W_{2}(\boldsymbol{p}, \boldsymbol{\gamma})}\left(t, \bar{\alpha}_{p}\right)-$ $r_{W_{2}(\boldsymbol{p}, \boldsymbol{\lambda})}\left(t, \bar{\alpha}_{p}\right)$.

\subsection{More counterexamples}

The following counterexamples can help in better understanding the limitations/assumptions of the considered in this section stochastic orderings. We first show that the result of Theorem 6.5 (Theorem 6.9) cannot be extended to the case $\boldsymbol{p} \stackrel{m}{\succeq} \boldsymbol{q}$.

Counterexample 6.23. Let $\bar{F}(t \mid \lambda)=e^{-\lambda t}$, for $t \in[0,+\infty)$. Clearly, $\bar{F}(t \mid \lambda)$ is decreasing and

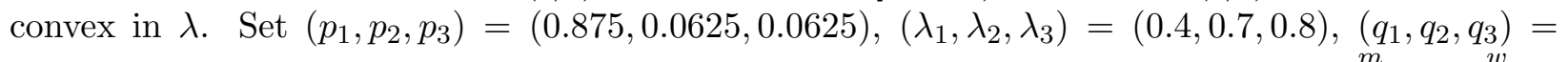
$(0.35,0.35,0.3),\left(\gamma_{1}, \gamma_{2}, \gamma_{3}\right)=(0.4,0.7,0.8),\left(\alpha_{1}, \alpha_{2}, \alpha_{3}\right)=(2.5,8,10)$. As we see, $\boldsymbol{p} \succeq \boldsymbol{q}, \boldsymbol{\lambda} \succeq \boldsymbol{w}$, $(\boldsymbol{p}, \boldsymbol{\lambda}) \in \mathcal{U}_{3}$ and $(\boldsymbol{q}, \boldsymbol{\gamma}) \in \mathcal{U}_{3}$. the plots in Figure 5 depict $\left.\left.\bar{F}_{W_{3}(\boldsymbol{p}, \boldsymbol{\lambda}}\right)\left(t, \bar{\alpha}_{p}\right), \bar{F}_{W_{3}(\boldsymbol{q}, \boldsymbol{\gamma}}\right)\left(t, \bar{\alpha}_{q}\right)$ and $\left.\left.g_{4}(t)=\bar{F}_{W_{3}(\boldsymbol{p}, \boldsymbol{\lambda}}\right)\left(t, \bar{\alpha}_{p}\right)-\bar{F}_{W_{3}(\boldsymbol{q}, \boldsymbol{\gamma}}\right)\left(t, \bar{\alpha}_{q}\right)$, which indicate that the usual stochastic ordering does not hold between $\left.\bar{F}_{W_{3}(\boldsymbol{p}, \boldsymbol{\lambda}}\right)\left(t, \bar{\alpha}_{p}\right)$ and $\left.\bar{F}_{W_{3}(\boldsymbol{q}, \boldsymbol{\gamma}}\right)\left(t, \bar{\alpha}_{q}\right)$.

In the following counterexample, we show that the usual stochastic ordering does not hold between $\bar{F}_{W_{n}(\boldsymbol{p}, \boldsymbol{\lambda})}\left(t, \bar{\alpha}_{p}\right)$ and $\bar{F}_{W_{n}(\boldsymbol{p}, \boldsymbol{\gamma})}\left(t, \bar{\beta}_{p}\right)$ when $\boldsymbol{\alpha} \succeq \stackrel{m}{\succeq} \boldsymbol{\beta}$.

Counterexample 6.24. Let $\bar{F}(t \mid \lambda)=e^{-\lambda t}$, for $t \in[0,+\infty)$. Clearly, $\bar{F}(t \mid \lambda)$ is decreasing. For $\alpha_{i} \geq 1$, set $\left(p_{1}, p_{2}, p_{3}\right)=(1 / 3,1 / 3,1 / 3),\left(\lambda_{1}, \lambda_{2}, \lambda_{3}\right)=(0.4,0.7,0.8),\left(\gamma_{1}, \gamma_{2}, \gamma_{3}\right)=(0.4,0.7,0.8)$ and $\left(\alpha_{1}, \alpha_{2}, \alpha_{3}\right)=(2.5,3.5,4)$ and $\left(\beta_{1}, \beta_{2}, \beta_{3}\right)=(2.8,3.4,3.8)$. As we see, $\boldsymbol{\lambda} \succeq \boldsymbol{\gamma},(\boldsymbol{p}, \boldsymbol{\lambda}) \in \mathcal{U}_{3}$ and $(\boldsymbol{p}, \boldsymbol{\gamma}) \in \mathcal{U}_{3}$ and $\boldsymbol{\alpha} \succeq \boldsymbol{m}$. Figure 6 (b) plots $g_{5}(t)=\bar{F}_{W_{3}(\boldsymbol{p}, \boldsymbol{\lambda})}\left(t, \bar{\alpha}_{p}\right)-\bar{F}_{W_{3}(\boldsymbol{p}, \boldsymbol{\gamma})}\left(t, \bar{\beta}_{p}\right)$ for $\alpha_{i}, \beta_{i} \geq 1$, which indicates that the usual stochastic ordering does not hold between $\left.\bar{F}_{W_{3}(\boldsymbol{p}, \boldsymbol{\lambda}}\right)\left(t, \bar{\alpha}_{p}\right)$ and $\left.\bar{F}_{W_{3}(\boldsymbol{p}, \boldsymbol{\gamma}}\right)\left(t, \bar{\beta}_{p}\right)$.

Finally, we demonstrate that the result of Theorem 6.17 cannot be extended to the likelihood ratio ordering 


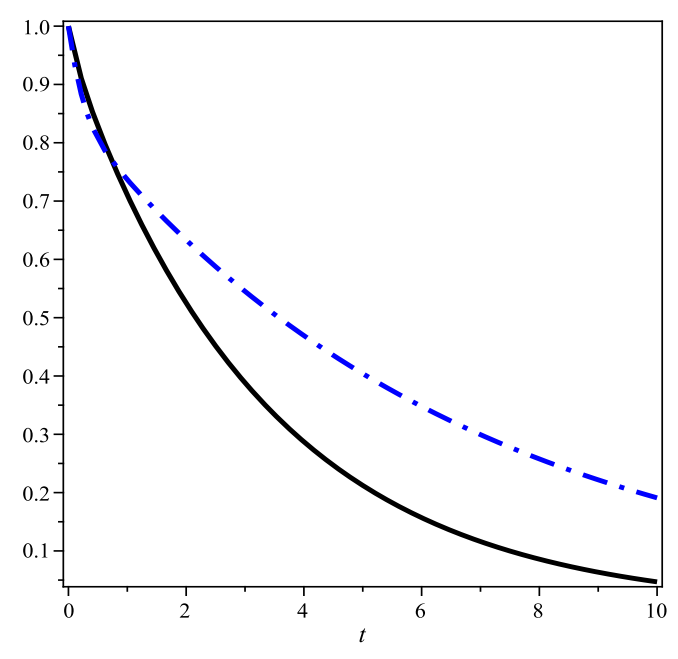

(a)

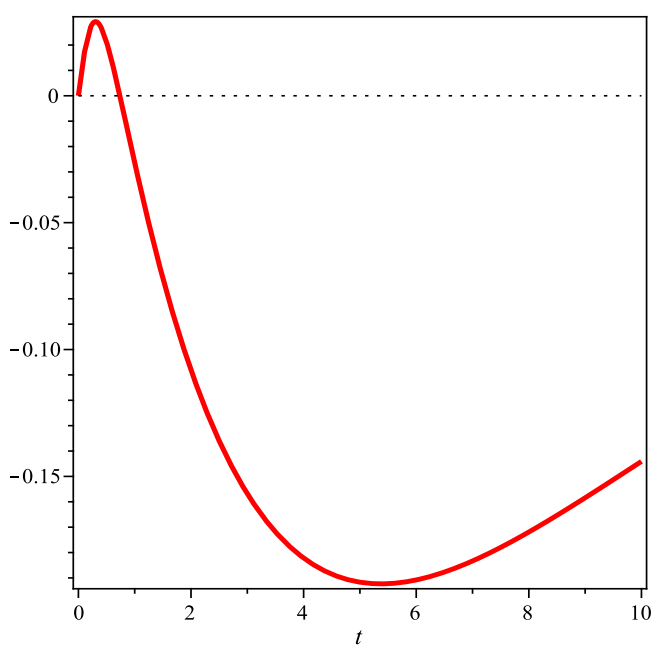

(b)

Figure 5: (a): $\left.\bar{F}_{W_{3}(\boldsymbol{p}, \boldsymbol{\lambda}}\right)\left(t, \bar{\alpha}_{p}\right)$ (solid) and $\left.\bar{F}_{W_{3}(\boldsymbol{q}, \boldsymbol{\gamma}}\right)\left(t, \bar{\alpha}_{p}\right)\left(\right.$ dash dot). (b): $\left.g_{4}(t)=\bar{F}_{W_{3}(\boldsymbol{p}, \boldsymbol{\lambda}}\right)\left(t, \bar{\alpha}_{p}\right)-$ $\left.\bar{F}_{W_{3}(\boldsymbol{q}, \gamma}\right)\left(t, \bar{\alpha}_{q}\right)$.

Counterexample 6.25. Let $\bar{F}(t \mid \lambda)=e^{-\lambda t}$, for $t \in[0,+\infty)$. Clearly, $\bar{F}(t \mid \lambda)$ is decreasing and $r(t \mid \lambda)=\lambda$ is increasing and concave in $\lambda$. For $\alpha_{i} \geq 0$, set $\left(p_{1}, p_{2}\right)=(0.55,0.45),\left(\lambda_{1}, \lambda_{2}\right)=(0.4,0.7)$, $\left(\gamma_{1}, \gamma_{2}\right)=(0.5,0.6)$ and $\left(\alpha_{1}, \alpha_{2}\right)=(2.5,3)$. As we see, $\boldsymbol{\lambda} \succeq \boldsymbol{m} \boldsymbol{\gamma},(\boldsymbol{p}, \boldsymbol{\lambda}) \in \mathcal{U}_{2}$ and $(\boldsymbol{p}, \boldsymbol{\gamma}) \in \mathcal{U}_{2}$ and $\left(\alpha_{1} p_{1}, \alpha_{2} p_{2}\right)=(1.375,1.35)$. Thus all conditions of Theorem 6.17 are satisfied. Figure 6 (a) plots $g_{6}(t)=\frac{f_{W_{2}(\boldsymbol{p}, \boldsymbol{\lambda})}\left(t, \bar{\alpha}_{p}\right)}{f_{W_{2}(\boldsymbol{p}, \boldsymbol{\gamma})}\left(t, \bar{\alpha}_{p}\right)}$ for $\alpha_{i} \geq 1$, which indicates that the likelihood ratio ordering does not hold between $\left.\bar{F}_{W_{2}(\boldsymbol{p}, \boldsymbol{\lambda}}\right)\left(t, \bar{\alpha}_{p}\right)$ and $\left.\bar{F}_{W_{2}(\boldsymbol{p}, \boldsymbol{\gamma}}\right)\left(t, \bar{\alpha}_{p}\right)$.

\section{Conclusions}

We have introduced the generalized finite $\alpha$-mixture of survival functions, which is a new flexible family of distributions that includes many mixture models as special cases and can be used to model, e.g., heterogeneity in populations. We have studied the hazard rate properties of the generalized finite $\alpha$-mixture family. Specifically, it was proved under some assumptions that the weakest (strongest) sub-population is smaller (greater) than the generalized finite $\alpha$-mixture in the sense of the hazard rate order. Moreover, an extension of the closure property of the $\alpha$-mixtures of DFR (IFR) distributions of Asadi et al. (2019) was provided, i.e., when all components are DFR (IFR) then the generalized finite $\alpha$-mixture is also DFR (IFR) under the specified assumptions. We also have showed that this closure property holds for DFRA (IFRA) distributions.

Some aging properties of the generalized finite $\alpha$-mixtures were investigated. In particular, for two important baseline hazard models, additive and multiplicative hazards, the hazard rate of the generalized finite $\alpha$-mixture is expressed in terms of the corresponding conditional moments of mixing distributions.

We have employed the concept of majorization for obtaining sufficient conditions for stochastic comparison of two generalized finite $\alpha$-mixture models in the sense of the usual stochastic order 


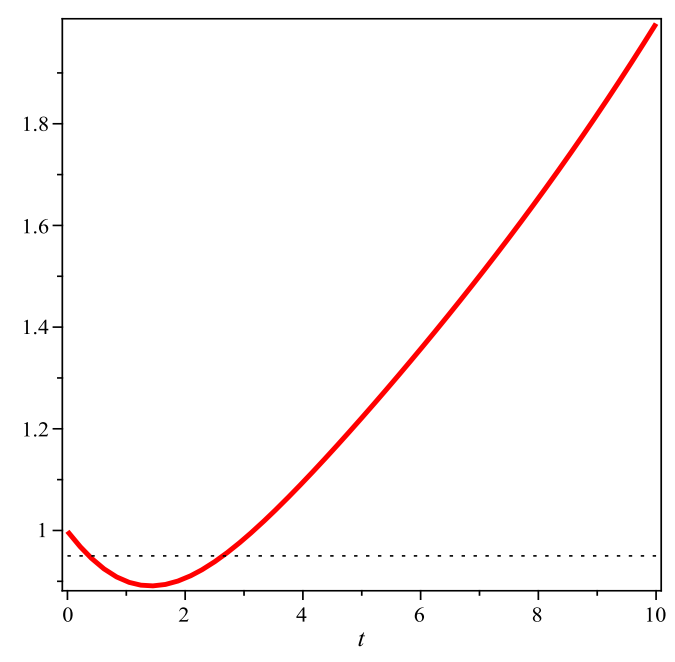

(a)

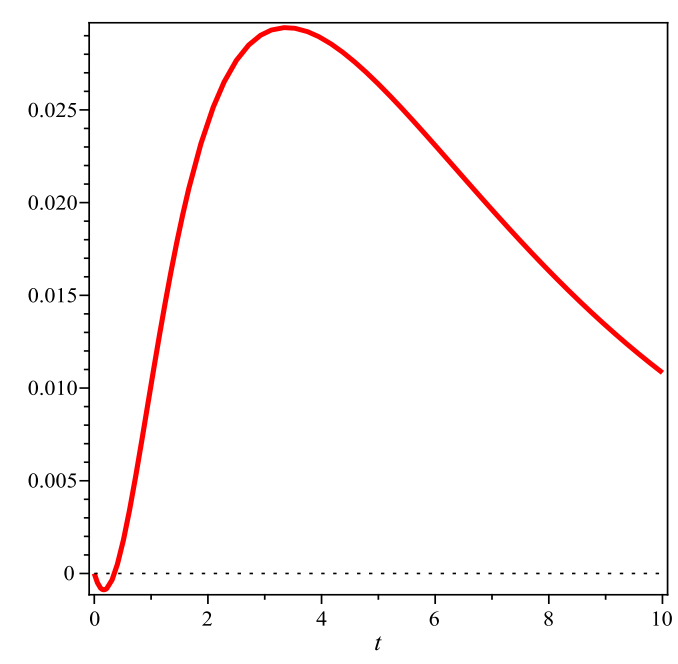

(b)

Figure 6: (a): $g_{6}(t)=\frac{f_{W_{2}(\boldsymbol{p}, \boldsymbol{\lambda})}\left(t, \bar{\alpha}_{p}\right)}{f_{W_{2}(\boldsymbol{p}, \boldsymbol{\gamma})}\left(t, \bar{\alpha}_{p}\right)} \cdot(\mathrm{b}): g_{5}(t)=\bar{F}_{W_{3}(\boldsymbol{p}, \boldsymbol{\lambda})}\left(t, \bar{\alpha}_{p}\right)-\bar{F}_{W_{3}(\boldsymbol{p}, \boldsymbol{\gamma})}\left(t, \bar{\beta}_{p}\right)$.

and the hazard rate order. The detailed analyses with numerous examples and counterexamples helps to understand the assumptions and limitations in our modeling.

\section{References}

[1] Albabtain, A. A., Shrahili, M., Al-Shehri, M. A. and Kayid, M. (2020). Stochastic comparisons of weighted distributions and their mixtures. Entropy, 22(8), 843.

[2] Amini-Seresht, E. and Zhang, Y. (2017). Stochastic comparisons on two finite mixture models. Operations Research Letters, 45(5), 475-480.

[3] Asadi, M., Ebrahimi, N. and Soofi, E. S. (2019). The alpha-mixture of survival functions. Journal of Applied Probability, 56(4), 1151-1167.

[4] Badia, F. G., Berrade, M. D. and Campos, C. A. (2002). Aging properties of the additive and proportional hazard mixing models. Reliability Engineering and System Safety, 78(2), 165-172.

[5] Badia, F. G., Berrade, M. D., Campos, C. A. and Navascues, M. A. (2001). On the behavior of aging characteristics in mixed populations. Probability in the Engineering and Informational Sciences, 15(1), 83-94.

[6] Badia, F. G. and Lee, H. (2020). On stochastic comparisons and ageing properties of multivariate proportional hazard rate mixtures. Metrika, 83(3), 355-375.

[7] Barlow, R. E. and Proschan, F. (1975). Statistical Theory of Reliability and Life Testing: Probability models. Florida State Univ Tallahassee. 
[8] Barmalzan, G., Kosari, S. and Zhang, Y. (2021). On stochastic comparisons of finite $\alpha$-mixture models. Statistics and Probability Letters, 173, 109083.

[9] Block, H. W. and Joe, H. (1997). Tail behavior of the failure rate functions of mixtures. Lifetime Data Analysis, 3(3), 269-288.

[10] Block, H. W., Li, Y. and Savits, T. H. (2003). Preservation of properties under mixture. Probability in the Engineering and Informational Sciences, 17(2), 205-212.

[11] Block, H. W. Mi, J. and Savits, T. H. (1993). Burn-in and mixed population. Journal of Applied probability, 30(3), 692-702.

[12] Block, H. W., Savits, T. H. and Wondmagegnehu, E. T. (2003). Mixtures of distributions with increasing linear failure rates. Journal of Applied Probability, 40, 485-504.

[13] Cha, J. H. (2011). Comparison of combined stochastic risk processes and its applications. European journal of operational research, 215(2), 404-410.

[14] Cha, J. H. and Finkelstein, M. (2013). The failure rate dynamics in heterogeneous populations. Reliability Engineering and System Safety, 112, 120-128.

[15] Everitt, B. S. and Hand, D. J. (1981). Finite mixture distributions. Chapman and Hall, New York.

[16] Finkelstein, M. (2008). Failure Rate Modelling for Reliability and Risk. Springer Science \& Business Media.

[17] Finkelstein, M. and Esaulova, V. (2001). Modeling a failure rate for a mixture of distribution function. Probability in the Engineering and Informational Sciences, 15(3), 383-400.

[18] Finkelstein, M. and Esaulova, V. (2006). On mixture failure rates ordering. Communications in Statistics - Theory and Methods, 35(11), 1943-1955.

[19] Hazra, N.K. and Finkelstein, M. (2018). On stochastic comparisons of finite mixtures for some semiparametric families of distributions. Test, 27, 988-1006.

[20] Hazra, N. K., Finkelstein, M. and Cha, J. H. (2017). On optimal grouping and stochastic comparisons for heterogeneous items. Journal of Multivariate Analysis, 160, 146-156.

[21] Marshall, A. W., Olkin, I. and Arnold, B. C. (2011). Inequalities: Theory of Majorization and its Applications. Springer, New York.

[22] Misra, N. and Naqvi, S. (2018). Stochastic comparison of residual lifetime mixture models. Operations Research Letters, 46(1), 122-127.

[23] Nadeb, H. and Torabi, H. (2020). New results on stochastic comparisons of finite mixtures for some families of distributions. Communications in Statistics-Theory and Methods, 1-16.

[24] Navarro, J. (2008). Likelihood ratio ordering of order statistics, mixtures and systems. Journal of Statistical Planning and Inference, 138(5), 1242-1257. 
[25] Navarro, J. (2016). Stochastic comparisons of generalized mixtures and coherent systems. Test, 25(1), 150-169.

[26] Navarro, J. and del Aguila, Y. (2017). Stochastic comparisons of distorted distributions, coherent systems and mixtures with ordered components. Metrika, 80(6-8), 627-648.

[27] Shaked, M. and Shanthikumar, J. G. (2007). Stochastic Orders. Springer Science \& Business Media.

[28] Shojaee, O., Asadi, M. and Finkelstein, M. (2021). On some properties of $\alpha$-mixtures. Metrika, $1-28$. 